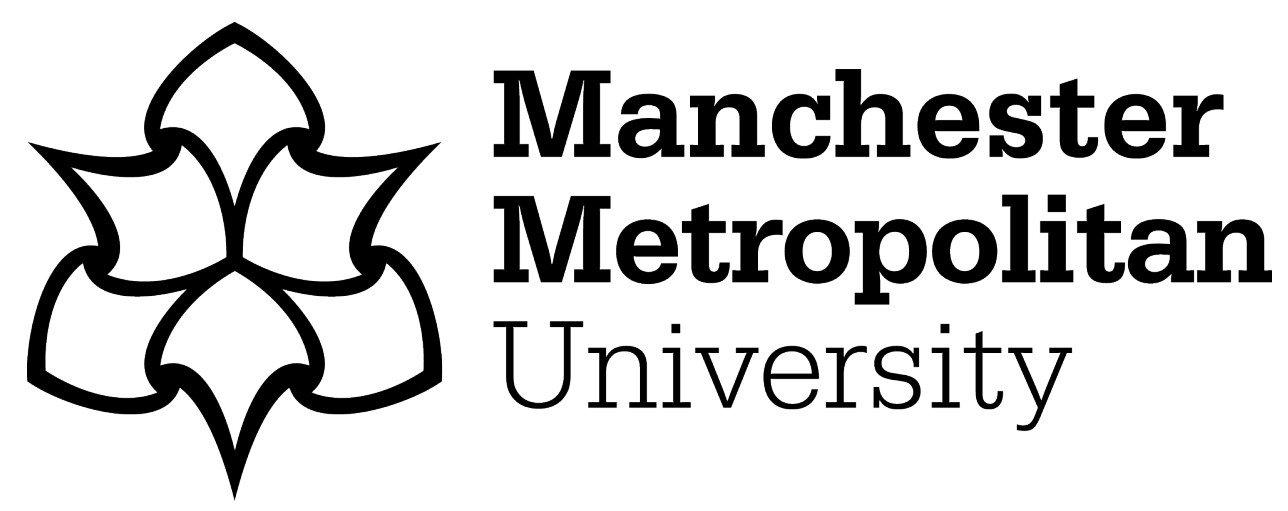

Basson, Ashley and McLaughlin, Mark Gerard (2020) Synthesis of Functionalized Isoindolinones via Calcium Catalyzed Generation and Trapping of NAcyliminium Ions. The Journal of Organic Chemistry, 85 (8). pp. 5615-5628. ISSN 0022-3263

Downloaded from: https://e-space.mmu.ac.uk/625429/

Version: Accepted Version

Publisher: American Chemical Society (ACS)

DOI: https://doi.org/10.1021/acs.joc.0c00482

Please cite the published version 


\title{
Article
}

\section{Synthesis of Functionalized Isoindolinones via Calcium Catalyzed Generation and Trapping of N-Acyliminium Ions}

\author{
Ashley Basson, and Mark Gerard McLaughlin
}

J. Org. Chem., Just Accepted Manuscript • DOI: 10.1021/acs.joc.0c00482 • Publication Date (Web): 25 Mar 2020

\section{Just Accepted}

"Just Accepted" manuscripts have been peer-reviewed and accepted for publication. They are posted online prior to technical editing, formatting for publication and author proofing. The American Chemical Society provides "Just Accepted" as a service to the research community to expedite the dissemination of scientific material as soon as possible after acceptance. "Just Accepted" manuscripts appear in full in PDF format accompanied by an HTML abstract. "Just Accepted" manuscripts have been fully peer reviewed, but should not be considered the official version of record. They are citable by the Digital Object Identifier (DOI®). "Just Accepted" is an optional service offered to authors. Therefore, the "Just Accepted" Web site may not include all articles that will be published in the journal. After a manuscript is technically edited and formatted, it will be removed from the "Just Accepted" Web site and published as an ASAP article. Note that technical editing may introduce minor changes to the manuscript text and/or graphics which could affect content, and all legal disclaimers and ethical guidelines that apply to the journal pertain. ACS cannot be held responsible for errors or consequences arising from the use of information contained in these "Just Accepted" manuscripts. 


\section{INTRODUCTION}

$N$-acyliminium ions represent a highly useful reactive intermediate that has found use in a plethora of synthetic methodologies and total synthesis alike. ${ }^{1-2}$ Over the course of several decades, these reactive intermediates have been employed to form new carbon-carbon and carbon-heteroatom bonds with great success. In particular, $N$-acyliminium ions have shown utility in the synthesis of fused ring systems through intramolecular trapping of the generated reactive intermediate. ${ }^{3-6}$ This strategy has been thoroughly explored over the last two decades with noted examples such as in the total syntheses of stemoamide, ${ }^{7}$ crispine $\mathrm{A},{ }^{8}$ and minfiensine (Fig 1). ${ }^{9}$

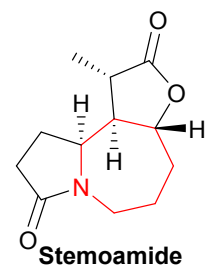

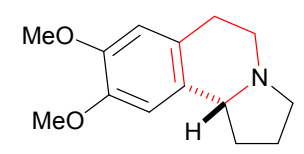

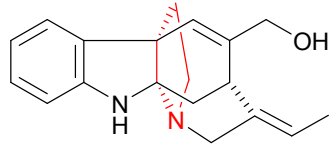

Crispine A

Minfiensine them using more functionally tolerant reagents remains high. Success using stoichiometric Lewis acids such as $\mathrm{BF}_{3} . \mathrm{OEt}_{2},{ }^{17}$ $\mathrm{Sc}(\mathrm{OTf})_{3}{ }^{18} \mathrm{TiCl}_{4},{ }^{19}$ and $\mathrm{AlCl}_{3}{ }^{20}$ have all recently been reported. Furthermore, organic acids such as $p$-TsOH and TFA have also been shown to generate $\mathrm{N}$-acyliminium ions efficiently under notably milder conditions. ${ }^{21}$ Although great successes have been described employing stoichiometric reagents, there is a clear need to develop catalytic processes by which these important intermediates can be generated. This need is twofold; firstly, catalytic generation inherently produces less waste, with the byproducts using hydroxyl or akyloxy leaving groups being water or innocuous alcohols. Secondly, from a reactivity point of view, catalytic generation will result in a more controlled reaction profile, with a much-reduced propensity for side reactions. Unsurprisingly, there has been noted successes in this endeavor (Scheme 1), with elegant examples using Brønsted

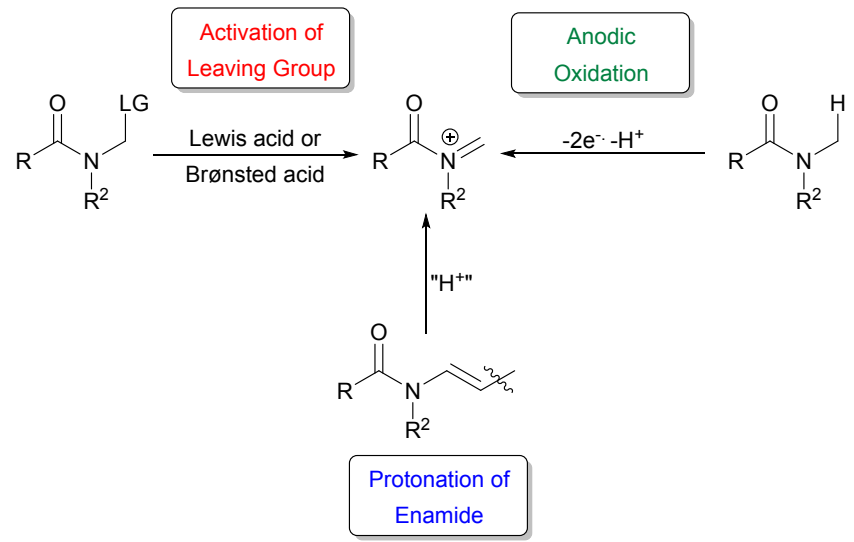

acids, ${ }^{22-24}$ thiourea organocatalysts ${ }^{25-28}$ and Lewis acids. ${ }^{29-33}$

Scheme 1 .Strategies towards N-acyliminium Ions based groups have also found use. ${ }^{16}$ As the synthetic utility of these intermediates becomes more obvious, the need to generate Traditionally, $N$-acyliminium ions are generated via the conditions. There is a wide range of these suitable leaving groups including hydroxyl, ${ }^{10}$ alkyl- and aryloxy, ${ }^{11}$ acetoxy ${ }^{12-13}$ and carbamate, ${ }^{14-15}$ however sulfur containing and sulfoxide 
Other methods including protonation of enamides ${ }^{34}$ and anodic (Shono)oxidation ${ }^{35}$ have also been described with varying success

Our group has a growing interest in developing methodology to catalytically generate reactive intermediates, ${ }^{36}$ and in particular using calcium complexes to mediate these processes. ${ }^{37-38}$ Calcium represents a relatively underexplored metal in catalysis, ${ }^{39}$ however over the past decade there has been an icrease in interest in exploring the reactivity of calcium, which has resulted in a wealth of innovative uses for this abundant element. ${ }^{40-60} \mathrm{We},{ }^{37}$ and others, ${ }^{61}$ have recently reported the use of $\mathrm{Ca}\left(\mathrm{NTf}_{2}\right)_{2}$ as an excellent catalyst to produce $N$-acyliminium ions from readily available 3-hydroxyisoindolinones. Due to the importance of these scaffolds in both total synthesis ${ }^{62-64}$ and medicinal chemistry, ${ }^{65}$ we wanted to explore this reaction further. In particular, we set out to probe the limits of the reaction towards traditional carbon, amine and sulfur nucleophiles. In particular, we wanted to focus our attention on the synthesis of functionalized isoindolinones, due to their importance in an ongoing medicinal chemistry campaign within our group. Unsurprisingly, due to their range of interesting biological activities, ${ }^{66-68}$ the synthesis of these scaffolds has attracted much attention in the literature (Scheme 2). In particular, the use of Brønsted and Lewis acids catalysts have been shown to be well tolerated. Although these examples provide the desired products in typically good yields, the use of tertiary hydroxyisoindolinones remains underexplored. This somewhat limits the use of the more complex catalytic systems, as it results in less than optimum substrate availability. Thus we wanted to explore these tertiary alcohols, and to probe the limits of the reactions.

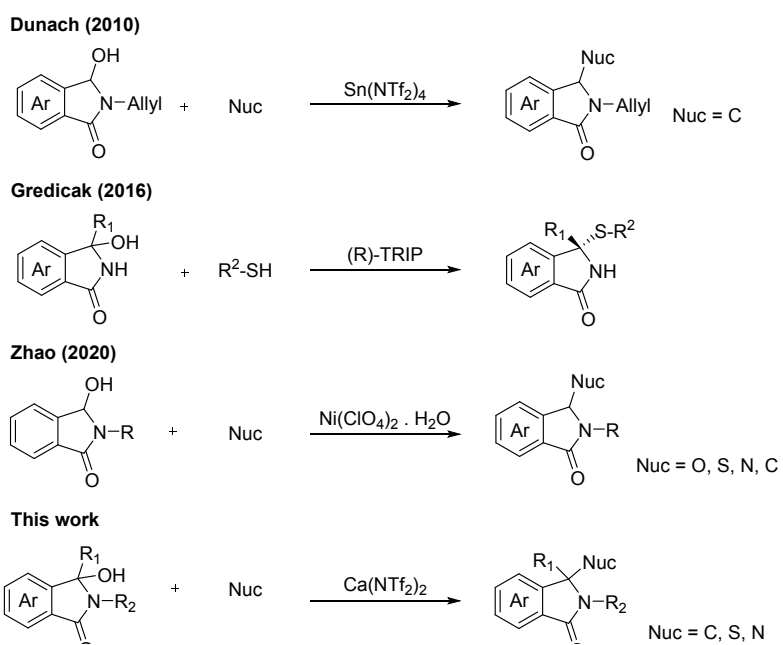

Scheme 2. Recent Successes in N-acyliminium chemistry

\section{RESULTS AND DISCUSSION}

We began our investigation employing indoles as nucleophiles. We rationalized that due to its importance in medicinal chemistry, paired with the availability of the motif, we could provide high value scaffolds bearing pendant functional groups in a controlled and high yielding manner. Furthermore, due to the ubiquitous nature of functionalized indoles in natural products, functionally tolerant catalytic methods to afford these types of compounds are constantly required. Indeed, upon treating 3-hydroxyisoindolinone with $10 \mathrm{~mol} \% \mathrm{Ca}\left(\mathrm{NTf}_{2}\right)_{2}$. $/ \mathrm{nBu}_{4} \mathrm{NPF}_{6}$ in the presence of 5-bromoindole, the desired product was formed in high yield (83\%). Reducing the catalyst loading to $1 \mathrm{~mol} \%$ had little effect on the reaction, providing the product in identical yield. Further optimization including varying the solvent, temperature and concentration had a deleterious effect on the yield of the reaction (Table 1). Finally, lowering the catalyst loading to below $1 \mathrm{~mol} \%$ resulted in a noticeably more sluggish reaction, and we decided that in the interest between balancing catalyst loading and reaction time, 1 mol\% was optimum. Importantly, we also performed a series of control experiments, most notable of which was using 2,6ditertbutylpyridine (entry 9), a known inhibitor of Brønsted acid catalysis. The reaction proceeded unhindered, and proves that the reaction is indeed calcium catalyzed.

\begin{tabular}{ccccc} 
& & & \\
\hline
\end{tabular}

a Isolated Yield b 2,6-ditertbutylpyridine added

\section{Table 1. Optimization study}

With these conditions in hand we probed the substrate scope of the reaction (Scheme 3). We observed excellent conversion to the products in all cases, providing a wide variety of complex scaffolds quickly. As shown, the reaction is tolerant to 5-bromo and 5-pinacolboronate indoles, affording a range of highly useful building blocks, including electron electron-withdrawing $(\mathbf{3 b})$ and donating groups $(\mathbf{3 c}, \mathbf{3 h})$ as well as further heterocyclic substrates $(\mathbf{3 d}, \mathbf{3 e}, \mathbf{3 i})$.

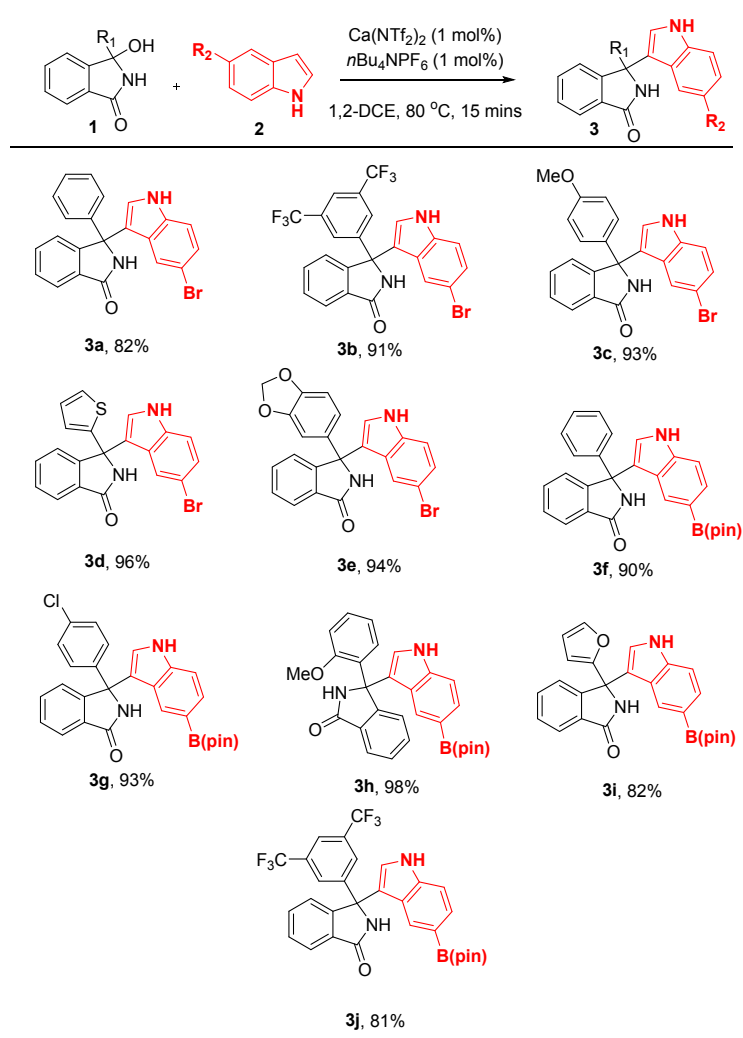

Scheme 3. Indole Substituted Isoindolinones 
As many catalytic processes are tolerant to carbon based functional groups, but show limited reactivity when heterocyclic reaction partners are used, we wanted explore the limits of the calcium system to these coupling partners. We decided to therefore move onto sulfur containing fragments, and in particular, aromatic and aliphatic thiols. $N($ acyl),$S-$ acetals are found in numerous pharmacologically relevant natural products and APIs alike. The most well-known of these being $\beta$-lactam antibiotics ${ }^{69}$ and HIV-1 Reverse Transcriptase inhibitors. ${ }^{67}$ Several methods have been developed for their synthesis, including a range of Brønsted acid catalyzed transformations,${ }^{70}$ however they typically require long reaction times and showed a limited scope towards certain functional groups. We reasoned that the use of calcium to mediate these reactions would have several advantages including much shorter reaction times, a wider functional group tolerance and overall easier reaction set up i.e. no requirement for anhydrous or air free condition. Traditionally, it would be expected that that the nucleophilic nature of these species would poison the catalyst, or at the very least, drastically hinder the catalytic turnover. However, to our delight, we found that the reaction using thiophenol proceeded well, with the reaction complete in under 15 minutes. Once again, we probed the substrate scope of the reaction, and a range of substituted hydroxyisoindolinones were subjected to our reaction conditions, resulting in a library of 3,3-disubstituted lactams (Scheme 4). As shown, the reaction was again tolerant of a range of functional groups with electron donating (4a-b) and withdrawing groups (4c-e) providing the desired products in high yields. Phenyl ring substituents was also well tolerated, with ortho and meta substituents proceeding smoothly (4f-g). Finally, heterocyclic substrates also worked well, providing the lactam products in high yields $(\mathbf{4 h}-\mathbf{j})$.

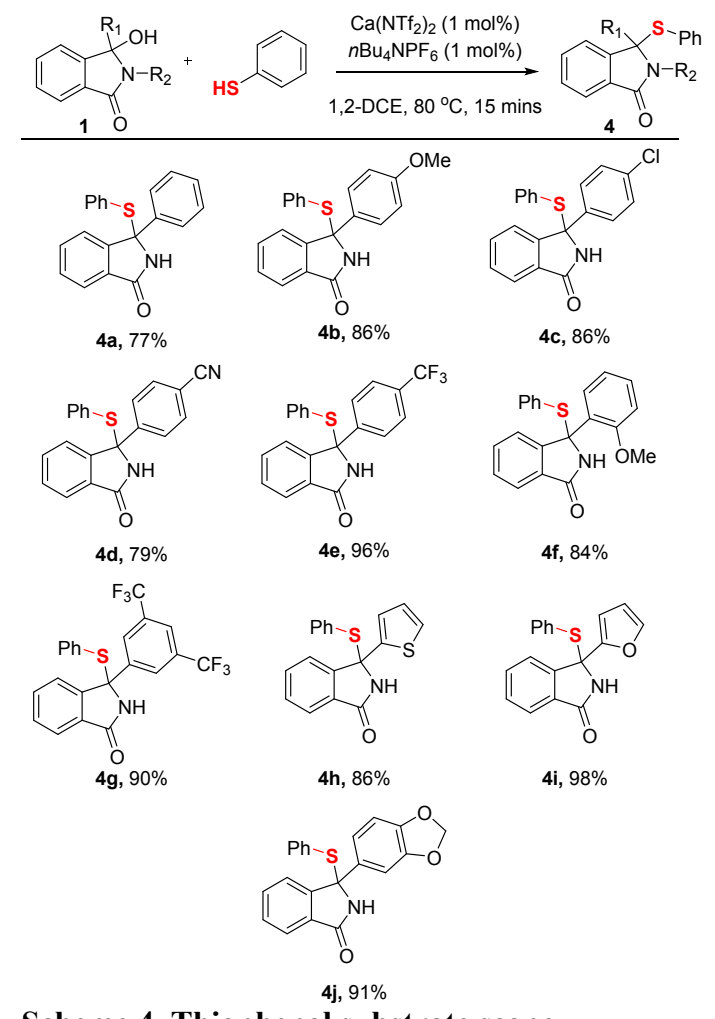

Scheme 4. Thiophenol substrate scope
As these examples all employed thiophenol as a nucleophile, we wanted to explore the tolerance of other sulfur derivatives. Once again, the reaction proceeded well, providing a wide range of medicinally relevant scaffolds (Scheme 5). In addition to the traditional electron donating $(\mathbf{6 a}, \mathbf{6 d}, \mathbf{6 e})$ and withdrawing $(\mathbf{6 b}$, 6c) groups, heteroaryl (6f, 6g), benzyl (6h) and alkyl $(\mathbf{6 i}, \mathbf{6 j})$ substituents all worked well. Furthermore, boronate esters (6k) are also well tolerated, providing avenues for the modular synthesis of bioactive small molecules.

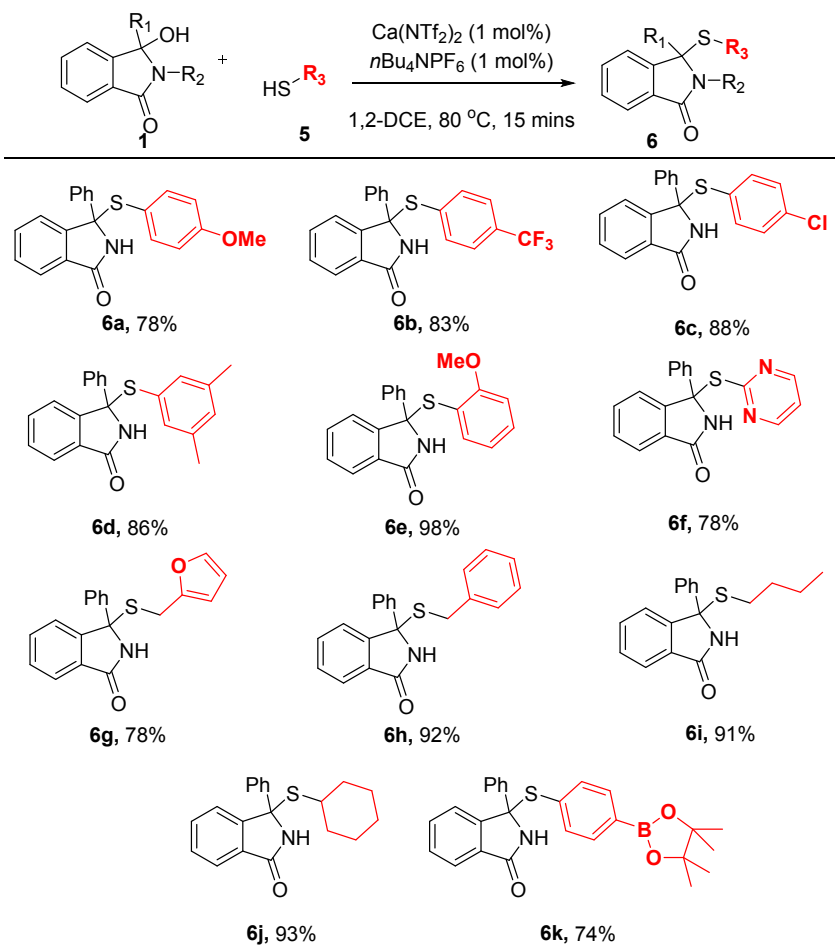

Scheme 5. Scope of Sulfur Nucleophiles

We next turned our attention to amine nucleophiles, as we envisaged that they would be more problematic in the reaction, mainly through direct Lewis acid-Lewis base interactions. Additionally, in contrast to their sulfur counterparts, 3aminoisoindolinones are relatively underexplored in synthesis, with very few reports focusing on the synthesis of carbamate and sulfonamide derivatives. This is somewhat surprising, given the fact that many isoindolinones have exhibited marked activity against a range of bacterial infections $\mathrm{s}^{71-72}$ as well as interesting anticancer properties. ${ }^{68}$ Due to this, we initially focused on delivering a robust method to produce these potentially high value molecules from readily available starting materials. We started our investigation employing our optimized conditions and simple amine nucleophiles (Scheme 6). We observed a clear trend in reactivity, in which only electron withdrawing anilines were tolerated, however we could never manage to isolate the products in useful yields. Additionally, the reaction was highly variable, and in our hands, remained unreproducible. On the small amount of material we did isolate, we observed a rapid degradation profile, with complete degradation after 2 hours, regardless of storage temperature. After some investigation, that the reaction employing N-Bn substituted hydroxyisoindolinones were much more user friendly, reproducibly providing the desired product in good yield. As shown, the reaction proceeded well, with substrates bearing contrasting electronics (8a-c) being tolerated. Furthermore, unsaturated $(\mathbf{8 d})$ and benzyl amines $(\mathbf{8 e})$ also worked well 


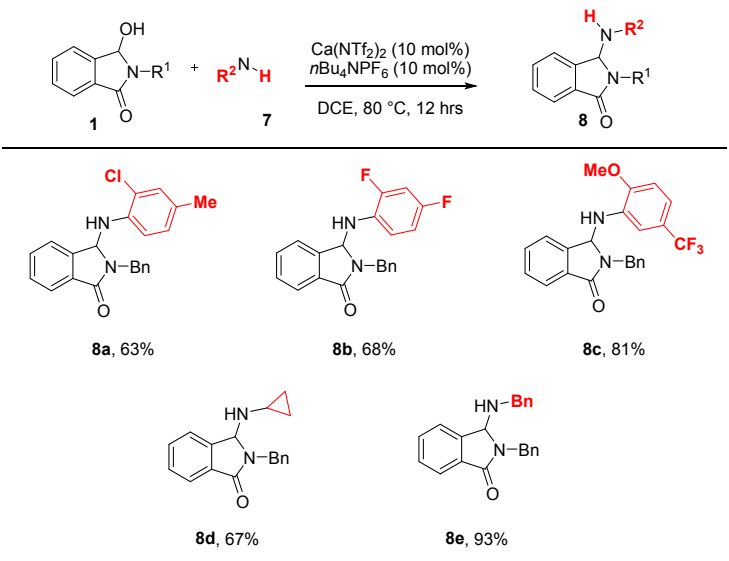

Scheme 6. 3-Amino substituted isoindolinones

Once complete, we probed the applicability of using less nucleophilic amine sources, such as the previously mentioned amide and sulfonamide moieties. Once again employing our optimized conditions, and using 1a as our model substrate, we screened a range of carbamate nucleophiles. Unfortunately, all of these proved unsuccessful, with the reactions plateauing at $25 \%$ conversion (Scheme 7).
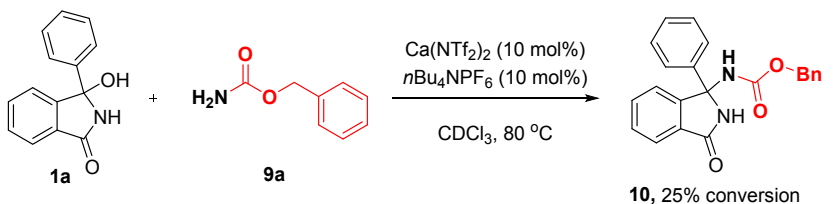

\section{Scheme 7. Reaction with Benzylcarbamate}

After extensive experimentation in which we screened a wide range of conditions including solvent, temperature, time and nucleophile, we were unsuccessful in delivering a reliable reaction that produced these compounds in synthetically useful yields. Although unsuccessful for compounds such as 1a, we were successful in performing the reaction on $\mathbf{1 1}$ using a small range of carbamate and amide nucleophiles, as shown (Scheme 8). This provides a range of differentially protected amines (12a-12c), which has the potential to find use in target synthesis.

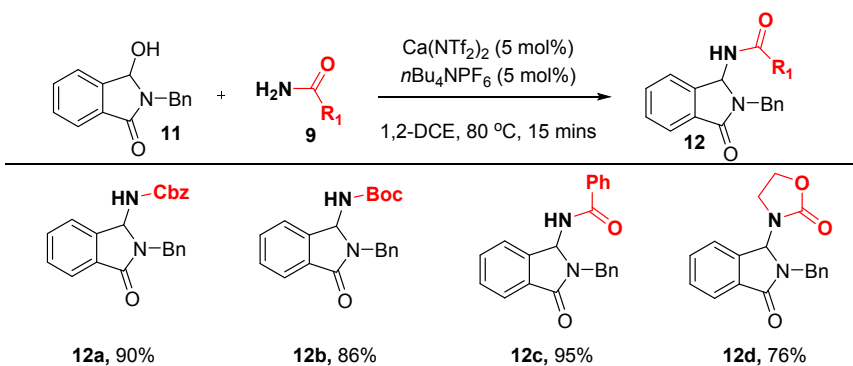

\section{Scheme 8. Carbamate and amide substitution reactions}

Due to the limited scope of these carbamates, we moved our attention to the more useful sulfonamides. Once again these have been seemingly ignored by the synthetic community, and therefore provides potentially new avenues of biological activities to be uncovered. The reactivity pattern remained, with only unsubstituted 11, providing reproducible reactivity and good yields throughout. Probing the variability of the sulfonamides produced a range of useful scaffolds using 5 mol\% of the catalyst system. As shown above (Scheme9), the reaction was once again tolerant to a range of synthetically useful functional groups including electron donating (14a, 14b) withdrawing (14c) halides (14d-f) thiophene (14g). Furthermore, saturated groups (14h) also worked well, providing access to 3 -dimensional scaffolds.

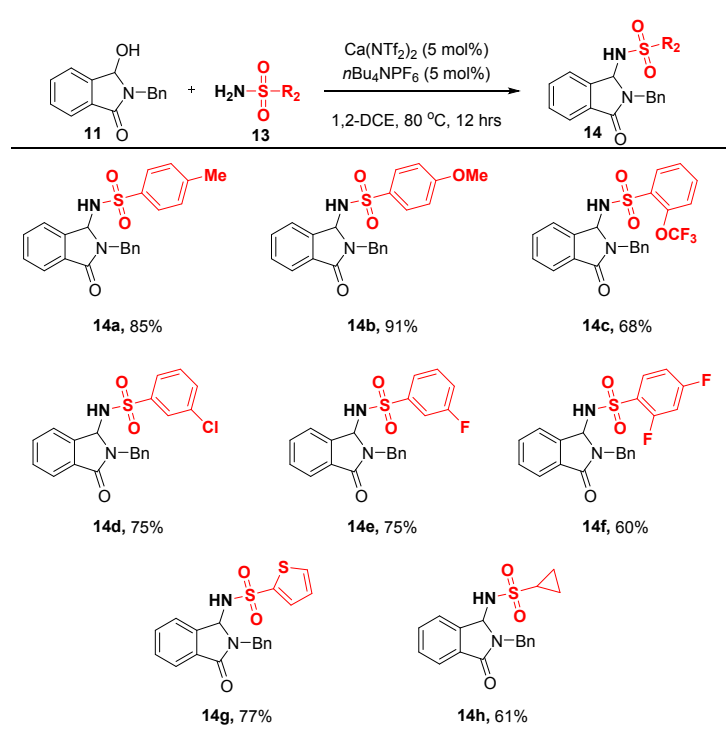

\section{Scheme 9. Functionalized isoindolinones bearing pendant sulfonamides}

As observed, the reaction is generally tolerant to a wide range of functional groups and nucleophiles. However, during the course of this work, we noted some limitations which we believe the community will find useful when deciding to use this methodology. Firstly, when employing amine nucleophiles, the reactions were very sluggish with tertiary alcohols such as 1a, with many reactions not going to completion. This was consistent across all amine nucleophiles used in this study. In a similar vein, we also observed that the choice of amine was important, with secondary amines such as piperidine and morpholine providing variable reactivity patterns, with irreproducible results being obtained. Secondly, carbon based nucleophiles (outside indole and allylsilane) were unreactive towards the conditions described here. We are currently working on this, and hope to report on it soon.

A proposed general mechanism is provided below (Fig. 2). The postulated active catalyst, $\left[\mathrm{CaPF}_{6} \mathrm{NTf}_{2}\right] \mathbf{A}$, is formed between $\mathrm{Ca}\left(\mathrm{NTf}_{2}\right)_{2}$ and $n \mathrm{Bu}_{4} \mathrm{NPF}_{6}$ which in turn produces $\mathrm{N}$ acyliminium ion $\mathbf{B}$ and complex $\mathbf{C}$ via loss of the noncoordinating $\mathrm{PF}_{6}$ ligand. Following nucleophilic attack, the aforementioned displaced $\mathrm{PF}_{6}$ re-enters the cycle, providing the product, water byproduct and reforming the active catalyst. 


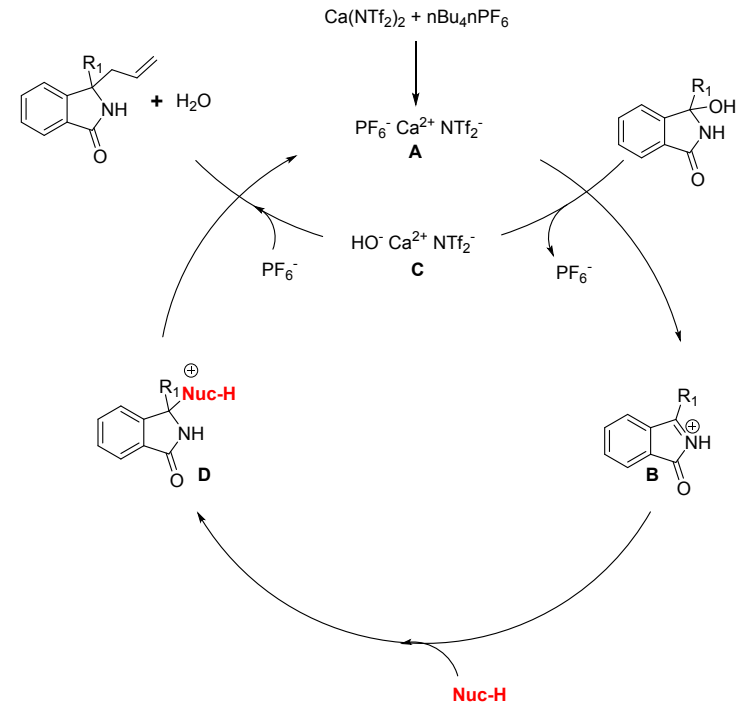

Figure 2. Proposed general catalytic cycle

In conclusion, we have developed a robust and high yield methodology to afford a range of substituted isoindolinones. Using low loadings of catalyst, the reaction proceeded smoothly, with reaction times ranging from 15 minutes to 12 hours. The reaction is tolerant towards a series of traditional nucleophiles including carbon, nitrogen and sulfur containing moieties. We envisage that this methodology will find use within medicinal chemistry campaigns due to the range of biological activities these scaffolds have shown.

\section{EXPERIMENTAL SECTION}

Solvents and Reagents. All solvents were purchased from commercial sources and used without purification (HPLC or analytical grade). Anhydrous solvent was obtained from a The Solv ${ }^{\mathrm{TM}}$ Solvent Purification System. Standard vacuum line techniques were used and glassware was oven dried prior to use. Organic solvents were dried during workup using anhydrous $\mathrm{Na}_{2} \mathrm{SO}_{4}$. All calcium catalyzed reactions where done without the need for anhydrous or air free conditions. All reaction were performed using DrySyn ${ }^{\mathrm{TM}}$ heating mantles and pressure regulated vials.

Purification and chromatography. Thin Layer Chromatography (TLC) was carried out using aluminum plates coated with 60 F254 silica gel. Plates were visualized using UV light (254 or $365 \mathrm{~nm}$ ) or staining with $1 \%$ aq. $\mathrm{KMnO}_{4}$, vanillin or ninhydrin. Normal-phase silica gel chromatography was carried out using either a Biotage Isolera One flash column chromatography system (LPLC) or traditional flash column chromatography using Geduran ${ }^{\circledR}$ Silica gel 60, 40-63 microns RE.

Characterization. Infrared spectroscopy was carried out with a Nicolet ${ }^{\circledR} 380$ FT/IR - Fourier Transform Infrared Spectrometer. Only the most significant frequencies have been considered during the characterization and selected absorption maxima (vmax) recorded in wavenumbers $\left(\mathrm{cm}^{-1}\right)$. NMR spectra were recorded using a JEOL ${ }^{\circledR}$ ECS-400 MHz spectrometer using the deuterated solvent stated. Chemical shifts $(\delta)$ quoted in parts per million (ppm) and referenced to the residual solvent peak. Multiplicities are denoted as s- singlet, d- doublet, ttriplet, q- quartet and quin- quintet and derivatives thereof (br denotes a broad resonance peak). Coupling constants recorded as $\mathrm{Hz}$ and round to the nearest $0.1 \mathrm{~Hz}$. High Resolution Mass Spectrometry (HRMS) was recorded using an Agilent Technologies ${ }^{\circledR} \quad 6540 \quad$ Ultra-High-Definition $\quad$ (UHD) AccurateMass equipped with a time of flight (Q-TOF) analyzer and the samples were ionized by ESI techniques and introduced through a high pressure liquid chromatography (HPLC) model Agilent Technologies ${ }^{\circledR} 1260$ Infinity Quaternary LC system.

3-(5-bromo-1H-indol-3-yl)-3-phenyl-2,3-dihydro-1H-isoindol1-one $(3 a)$. To a $4 \mathrm{~mL}$ vial was added 3-hydroxy-3phenylisoindolin-1-one $(50 \mathrm{mg}, 0.22 \mathrm{mmol}), \mathrm{Ca}\left(\mathrm{NTf}_{2}\right)_{2}(1.3$ $\mathrm{mg}, 0.0022 \mathrm{mmol})$ and $n \mathrm{Bu}_{4} \mathrm{NPF}_{6}(1 \mathrm{mg}, 0.0022 \mathrm{mmol})$ in DCE $(1 \mathrm{~mL})$ at $80{ }^{\circ} \mathrm{C}$. The reaction was stirred for 1 minute and 5 bromoindole $(65 \mathrm{mg}, 0.33 \mathrm{mmol})$ was added in a single portion and stirred at $80^{\circ} \mathrm{C}$ for 15 minutes. Once TLC analysis indicated conversion to the product, the product was concentrated and purified by flash column chromatography (3:1 EtOAc:Hex) to afford a cream solid $(73 \mathrm{mg}, 82 \%)$. RF $(3: 1 \mathrm{EtOAc} / \mathrm{Hex})=0.37$. IR $v_{\max }\left(\mathrm{cm}^{-1}\right): 3209,3058,1978,1671,1491,1314$. HRMS (ESI) $\mathrm{m} / \mathrm{z}$ : $[\mathrm{M}+\mathrm{H}]^{+}$Calcd for $\mathrm{C}_{22} \mathrm{H}_{16} \mathrm{BrN}_{2} \mathrm{O}$ 403.0446; found 403.0441.. ${ }^{1} \mathrm{H}$ NMR (400 MHz, DMSO-d ${ }_{6}$ ): $\delta 11.30(\mathrm{~s}, 1 \mathrm{H})$, $9.73(\mathrm{~s}, 1 \mathrm{H}), 7.74(\mathrm{~d}, J=7.4 \mathrm{~Hz}, 1 \mathrm{H}), 7.64-7.57(\mathrm{~m}, 2 \mathrm{H}), 7.53$ $(\mathrm{dq}, J=4.7,4.1 \mathrm{~Hz}, 1 \mathrm{H}), 7.45(\mathrm{~d}, J=7.2 \mathrm{~Hz}, 2 \mathrm{H}), 7.40-7.25$ (m, 4H), 7.16 (dd, $J=8.6,1.6 \mathrm{~Hz}, 1 \mathrm{H}), 6.94(\mathrm{dd}, J=15.7,1.8$ $\mathrm{Hz}, 2 \mathrm{H}) .{ }^{13} \mathrm{C}\left\{{ }^{1} \mathrm{H}\right\}\left(101 \mathrm{MHz}, \mathrm{DMSO}-\mathrm{d}_{6}\right): \delta 168.6,150.6,142.5$, $135.8,132.1,130.9,128.5,127.6,126.9,126.3,125.9,124.2$, $123.9,123.3,122.1,116.5,113.8,111.4,65.9$.

Reaction also performed on a $2.2 \mathrm{mmol}$ from 3-hydroxy-3phenylisoindolin-1-one (500 mg, $2.20 \mathrm{mmol}), \mathrm{Ca}\left(\mathrm{NTf}_{2}\right)_{2}(13$ $\mathrm{mg}, 0.02 \mathrm{mmol})$ and $n \mathrm{Bu}_{4} \mathrm{NPF}_{6}(9 \mathrm{mg}, 0.02 \mathrm{mmol})$ with 5 bromoindole $(653 \mathrm{mg}, 3.33 \mathrm{mmol})$ in DCE $(10 \mathrm{~mL})$ for $2.5 \mathrm{~h}$. Purification (3:1 EtOAc:Hex) afforded the product as a cream solid (880 mg, 98\%)

3-[3,5-bis(trifluoromethyl)phenyl]-3-(5-bromo-1H-indol-3-yl)2,3-dihydro-1H-isoindol-1-one (3b). To a $4 \mathrm{~mL}$ vial was added 3-hydroxy-3-(3,5-bis(trifluoromethyl))isoindolin-1-one (50 $\mathrm{mg}, 0.14 \mathrm{mmol}), \mathrm{Ca}\left(\mathrm{NTf}_{2}\right)_{2}(4 \mathrm{mg}, 0.0069 \mathrm{mmol})$ and $n \mathrm{Bu}_{4} \mathrm{NPF}_{6}(3 \mathrm{mg}, 0.0069 \mathrm{mmol})$ in DCE $(1 \mathrm{~mL})$ at $80{ }^{\circ} \mathrm{C}$. The reaction was stirred for 1 minute and 5-bromoindole $(41 \mathrm{mg}$, $0.21 \mathrm{mmol}$ ) was added in a single portion and stirred at $80^{\circ} \mathrm{C}$ for 30 minutes. Once TLC analysis indicated conversion to the product, the product was concentrated and purified by flash column chromatography $(1: 1$, EtOAC:Hex $)$ to afford a cream solid $(68 \mathrm{mg}, 91 \%)$. RF $(1: 1 \mathrm{EtOAc} / \mathrm{Hex})=0.33 . \mathrm{IR} v_{\max }\left(\mathrm{cm}^{-}\right.$ $\left.{ }^{1}\right): 3333,3185,3052,1978,1669,1368$. HRMS (ESI) m/z: $[\mathrm{M}+\mathrm{H}]^{+}$Calcd for $\mathrm{C}_{24} \mathrm{H}_{14} \mathrm{BrF}_{6} \mathrm{~N}_{2} \mathrm{O}$ 539.0194; found 539.0188. ${ }^{1} \mathrm{H}$ NMR $\left(400 \mathrm{MHz}, \mathrm{DMSO}-\mathrm{d}_{6}\right): \delta 11.44(\mathrm{~s}, 1 \mathrm{H}), 9.91(\mathrm{~s}, 1 \mathrm{H})$ $8.16(\mathrm{~s}, 1 \mathrm{H}), 8.11(\mathrm{~s}, 2 \mathrm{H}), 7.79(\mathrm{dd}, J=15.3,7.5 \mathrm{~Hz}, 2 \mathrm{H}), 7.67$ (t, $J=7.4 \mathrm{~Hz}, 1 \mathrm{H}), 7.60(\mathrm{t}, J=7.4 \mathrm{~Hz}, 1 \mathrm{H}), 7.38(\mathrm{~d}, J=8.6 \mathrm{~Hz}$, $1 \mathrm{H}), 7.19(\mathrm{dd}, J=8.7,1.3 \mathrm{~Hz}, 1 \mathrm{H}), 6.98(\mathrm{~d}, J=2.4 \mathrm{~Hz}, 1 \mathrm{H})$, $6.83(\mathrm{~s}, 1 \mathrm{H}) .{ }^{13} \mathrm{C}\left\{{ }^{1} \mathrm{H}\right\}$ NMR $\left(101 \mathrm{MHz}, \mathrm{DMSO}-\mathrm{d}_{6}\right): \delta 168.4$, $149.0,145.9,135.8,132.8,130.8,130.5\left(\mathrm{q}, J_{F}=32.3 \mathrm{~Hz}\right)$, $129.3,127.0,126.9,126.3,124.6,124.3,124.2,123.6$, $122.1,121.8,121.4,115.1,114.1,111.7,65.5$.

3-(5-bromo-1H-indol-3-yl)-3-(4-methoxyphenyl)-2,3-dihydro1 -isoindol-1-one $(3 \mathrm{c})$. To a $4 \mathrm{~mL}$ vial was added 3-(4methoxyphenyl)-3-hydroxyisoindolin-1-one $(50 \mathrm{mg}, \quad 0.20$ $\mathrm{mmol} \mathrm{mmol}), \mathrm{Ca}\left(\mathrm{NTf}_{2}\right)_{2}(1.1 \mathrm{mg}, 0.002 \mathrm{mmol})$ and $n \mathrm{Bu}_{4} \mathrm{NPF}_{6}$ $(0.8 \mathrm{mg}, 0.002 \mathrm{mmol})$ in DCE $(1 \mathrm{~mL})$ at $80{ }^{\circ} \mathrm{C}$. The reaction was stirred for 1 minute and 5-bromoindole $(58 \mathrm{mg}, 0.29 \mathrm{mmol})$ 
was added in a single portion and stirred at $80^{\circ} \mathrm{C}$ for 30 minutes. Once TLC analysis indicated conversion to the product, the product was concentrated and purified by flash column chromatography (1:1, EtOAc:Hex) to afford a cream solid (79 $\mathrm{mg}, 93 \%)$. RF $(1: 1 \mathrm{EtOAc} / \mathrm{DCM})=0.41 . \mathrm{IR} \mathrm{v}_{\max }\left(\mathrm{cm}^{-1}\right): 3169$, 3040, 2838, 1668, 1608, 1255. HRMS (ESI) m/z: $[\mathrm{M}+\mathrm{H}]^{+}$ Calcd for $\mathrm{C}_{23} \mathrm{H}_{18} \mathrm{BrN}_{2} \mathrm{O}_{2} 433.0552$; ; found 433.0549.. ${ }^{1} \mathrm{H}$ NMR (400 MHz, DMSO-d $\left.)_{6}\right): \delta 11.28(\mathrm{~s}, 1 \mathrm{H}), 9.67(\mathrm{~s}, 1 \mathrm{H}), 7.73(\mathrm{~d}, J$ $=7.3 \mathrm{~Hz}, 1 \mathrm{H}), 7.65-7.46(\mathrm{~m}, 3 \mathrm{H}), 7.34(\mathrm{t}, J=8.6 \mathrm{~Hz}, 3 \mathrm{H})$, $7.16(\mathrm{~d}, J=8.5 \mathrm{~Hz}, 1 \mathrm{H}), 7.01(\mathrm{~s}, 1 \mathrm{H}), 6.99-6.79(\mathrm{~m}, 3 \mathrm{H}), 3.72$ (s, 3H). ${ }^{13} \mathrm{C}\left\{{ }^{1} \mathrm{H}\right\}$ NMR $(101 \mathrm{MHz}$, DMSO-d 6 ): $\delta 168.5,158.6$, $150.9,135.8,134.4,132.0,130.9,128.4,127.6,126.9,125.9$, $124.1,123.9,123.2,122.2,116.7,113.8,113.7,111.4,65.50$, 55.11 .

3-(5-bromo-1H-indol-3-yl)-3-(thiophen-2-yl)-2,3-dihydro-1Hisoindol-1-one $(\mathbf{3 d})$. To a $4 \mathrm{~mL}$ vial was added 3-hydroxy-3(thiophen-2-yl)isoindolin-1-one $\quad(50 \mathrm{mg}, 0.22 \mathrm{mmol})$, $\mathrm{Ca}\left(\mathrm{NTf}_{2}\right)_{2}(1.3 \mathrm{mg}, 0.0022 \mathrm{mmol})$ and $n \mathrm{Bu}_{4} \mathrm{NPF}_{6}(0.9 \mathrm{mg}$, $0.0022 \mathrm{mmol})$ in DCE $(1 \mathrm{~mL})$ at $80^{\circ} \mathrm{C}$. The reaction was stirred for 1 minute and 5-bromoindole $(64 \mathrm{mg}, 0.32 \mathrm{mmol})$ was added in a single portion and stirred at $80{ }^{\circ} \mathrm{C}$ for 30 minutes. Once TLC analysis indicated conversion to the product, the product was concentrated and purified by flash column chromatography (1:1 EtOAc:DCM) to afford a light brown solid (85 $\mathrm{mg}, 96 \%)$. $\mathrm{RF}(1: 1 \mathrm{EtOAc} / \mathrm{DCM})=0.38$. IR $v_{\max }\left(\mathrm{cm}^{-1}\right): 3172,3041,2851$, 1667, 1466, 1356. HRMS (ESI) $\mathrm{m} / \mathrm{z}:[\mathrm{M}+\mathrm{H}]^{+}$Calcd for $\mathrm{C}_{20} \mathrm{H}_{14} \mathrm{BrN}_{2} \mathrm{OS} 409.0010$; found 409.0007. Cald. ${ }^{1} \mathrm{H}$ NMR (400 MHz, DMSO-d $\left.{ }_{6}\right): \delta 11.36(\mathrm{~m}, 1 \mathrm{H}), 9.85(\mathrm{~s}, 1 \mathrm{H}), 7.76(\mathrm{~d}, J=7.4$ $\mathrm{Hz}, 1 \mathrm{H}), 7.68-7.51(\mathrm{~m}, 3 \mathrm{H}), 7.45(\mathrm{~d}, J=5.0 \mathrm{~Hz}, 1 \mathrm{H}), 7.35(\mathrm{~d}$, $J=8.6 \mathrm{~Hz}, 1 \mathrm{H}), 7.17(\mathrm{dd}, J=8.6,1.6 \mathrm{~Hz}, 1 \mathrm{H}), 7.08(\mathrm{~m}, 2 \mathrm{H})$, $7.05-6.92(\mathrm{~m}, 2 \mathrm{H}) \cdot{ }^{13} \mathrm{C}\left\{{ }^{1} \mathrm{H}\right\}$ NMR $\left(101 \mathrm{MHz}, \mathrm{DMSO}-\mathrm{d}_{6}\right): \delta$ $168.3,150.6,147.3,135.7,132.3,130.5,128.8,127.2,126.7$, $126.0,125.7,125.5,124.0,123.9,123.2,121.9,115.4,113.9$, $111.6,63.53$.

3-(2H-1,3-benzodioxol-5-yl)-3-(5-bromo-3a,7a-dihydro-1Hindol-2-yl)-2,3-dihydro-1H-isoindol-1-one (3e). To a $4 \mathrm{~mL}$ vial was added 3-(benzo[d][1,3]dioxol-5-yl)isoindolin-1-one (50 $\mathrm{mg}, 0.19 \mathrm{mmol}), \mathrm{Ca}\left(\mathrm{NTf}_{2}\right)_{2}(1.1 \mathrm{mg}, 0.0019 \mathrm{mmol})$ and $n \mathrm{Bu}_{4} \mathrm{NPF}_{6}(0.7 \mathrm{mg}, 0.0019 \mathrm{mmol})$ in DCE $(1 \mathrm{~mL})$ at $80^{\circ} \mathrm{C}$. The reaction was stirred for 1 minute and 5-bromoindole $(64 \mathrm{mg}$, $0.32 \mathrm{mmol}$ ) was added in a single portion and stirred at $80^{\circ} \mathrm{C}$ for 30 minutes. Once TLC analysis indicated conversion to the product, the product was concentrated and purified by flash column chromatography $(2: 1$, EtOAc:Hex $)$ to afford a cream solid $(78 \mathrm{mg}, 94 \%)$. RF $(2: 1 \mathrm{EtOAc} / \mathrm{Hex})=0.17$. IR $\mathrm{v}_{\max }\left(\mathrm{cm}^{-}\right.$ $\left.{ }^{1}\right)$ : 3219, 2896, 1674, 1611, 1485, 1237. HRMS (ESI) m/z: $[\mathrm{M}+\mathrm{H}]^{+}$Calcd for $\mathrm{C}_{23} \mathrm{H}_{16} \mathrm{BrN}_{2} \mathrm{O}_{3} 447.0344$; found 447.0350. ${ }^{1} \mathrm{H}$ NMR $\left(400 \mathrm{MHz}, \mathrm{CDCl}_{3}\right): \delta 8.25(\mathrm{~s}, 1 \mathrm{H}), 7.89(\mathrm{~d}, J=7.4 \mathrm{~Hz}$, $1 \mathrm{H}), 7.63-7.40(\mathrm{~m}, 3 \mathrm{H}), 7.31-7.21(\mathrm{~m}, 2 \mathrm{H}), 7.16(\mathrm{~s}, 1 \mathrm{H}), 6.96$ $-6.85(\mathrm{~m}, 3 \mathrm{H}), 6.82(\mathrm{~d}, J=1.8 \mathrm{~Hz}, 1 \mathrm{H}), 6.72(\mathrm{~d}, J=8.2 \mathrm{~Hz}$, $1 \mathrm{H}), 5.95$ (dd, $J=6.6,1.1 \mathrm{~Hz}, 2 \mathrm{H}) .{ }^{13} \mathrm{C}\left\{{ }^{1} \mathrm{H}\right\} \mathrm{NMR}(101 \mathrm{MHz}$, $\left.\mathrm{CDCl}_{3}\right): \delta 169.9,150.9,148.3,147.7,135.9,135.3,132.6$, $128.8,127.3,125.9,124.6,124.3,123.7,122.7,119.9,117.7$, $113.7,113.3,108.5,107.2,101.5,66.5$.

3-phenyl-3-[5-(4,4,5,5-tetramethyl-1,3,2-dioxaborolan-2-yl)3a,7a-dihydro-1H-indol-2-yl]-2,3-dihydro-1H-isoindol-1-one (3f). To a $4 \mathrm{~mL}$ vial was added 3-hydroxy-3-phenylisoindolin1-one (50 mg, $0.22 \mathrm{mmol}), \mathrm{Ca}\left(\mathrm{NTf}_{2}\right)_{2}(1.3 \mathrm{mg}, 0.0022 \mathrm{mmol})$ and $n \mathrm{Bu}_{4} \mathrm{NPF}_{6}(0.9 \mathrm{mg}, 0.0022 \mathrm{mmol})$ in DCE $(1 \mathrm{~mL})$ at $80^{\circ} \mathrm{C}$. The reaction was stirred for 1 minute and 5-(4,4,5,5Tetramethyl-1,3,2-dioxaborolan-2-yl)-1H-indole (81 mg, 0.33 mmol) was added in a single portion and stirred at $80^{\circ} \mathrm{C}$ for $1 \mathrm{~h}$. Once TLC analysis indicated conversion to the product, the product was concentrated and purified by flash column chromatography $(2: 1$, EtOAc:Hex) to afford a white solid (90 $\mathrm{mg}, 90 \%)$. RF (3:1 EtOAc/Hex) $=0.48$. IR $v_{\max }\left(\mathrm{cm}^{-1}\right): 3257$, 2976, 1683, 1613, 1352, 1142. HRMS (ESI) m/z: $[\mathrm{M}+\mathrm{H}]^{+}$ Calcd for $\mathrm{C}_{28} \mathrm{H}_{28} \mathrm{BN}_{2} \mathrm{O}_{3}$ 451.2193; found 451.2204. ${ }^{1} \mathrm{H}$ NMR $\left(400 \mathrm{MHz}, \mathrm{DMSO}-\mathrm{d}_{6}\right): \delta 11.20(\mathrm{~s}, 1 \mathrm{H}), 9.73(\mathrm{~s}, 1 \mathrm{H}), 7.74(\mathrm{~d}, J$ $=7.3 \mathrm{~Hz}, 1 \mathrm{H}), 7.65-7.44(\mathrm{~m}, 4 \mathrm{H}), 7.44-7.22(\mathrm{~m}, 7 \mathrm{H}), 6.88$ $(\mathrm{d}, J=2.2 \mathrm{~Hz}, 1 \mathrm{H}), 1.22(\mathrm{~s}, 12 \mathrm{H}) .{ }^{13} \mathrm{C}\left\{{ }^{1} \mathrm{H}\right\} \mathrm{NMR}(101 \mathrm{MHz}$, DMSO-d ${ }_{6}$ ): $\delta 168.6,151.1,142.9,139.2,131.9,131.0,128.4$, $128.3,127.7,127.5,127.5,126.5,124.8,124.3,124.2,123.2$, $117.2,111.2,83.0,66.2,24.8,24.6$.

3-(4-chlorophenyl)-3-[5-(4,4,5,5-tetramethyl-1,3,2dioxaborolan-2-yl)-3a,7a-dihydro-1H-indol-2-yl]-2,3-dihydro1H-isoindol-1-one (3g). To a $4 \mathrm{~mL}$ vial was added 3-(4chlorophenyl)-3-hydroxyisoindolin-1-one (50 mg, $0.19 \mathrm{mmol})$, $\mathrm{Ca}\left(\mathrm{NTf}_{2}\right)_{2}(1 \mathrm{mg}, 0.0019 \mathrm{mmol})$ and $n \mathrm{Bu}_{4} \mathrm{NPF}_{6}(0.8 \mathrm{mg}, 0.0019$ $\mathrm{mmol})$ in DCE $(1 \mathrm{~mL})$ at $80^{\circ} \mathrm{C}$. The reaction was stirred for 1 minute and 5-(4,4,5,5-Tetramethyl-1,3,2-dioxaborolan-2-yl)$1 \mathrm{H}$-indole $(70 \mathrm{mg}, 0.29 \mathrm{mmol})$ was added in a single portion and stirred at $80{ }^{\circ} \mathrm{C}$ for 30 mins. Once TLC analysis indicated conversion to the product, the product was concentrated and purified by flash column chromatography $(1: 1$, EtOAc:Hex $)$ to afford a white solid (87 mg, 93\%). RF (1:1 EtOAc/Hex $)=0.19$. IR $v_{\max }\left(\mathrm{cm}^{-1}\right): 3237,2977,1673,1613,1469,1352$. HRMS (ESI) $\mathrm{m} / \mathrm{z}$ : $[\mathrm{M}+\mathrm{H}]^{+}$Calcd for $\mathrm{C}_{28} \mathrm{H}_{27} \mathrm{BCIN}_{2} \mathrm{O}_{3}$ 485.1803; found 485.1815. . ${ }^{1} \mathrm{H}$ NMR (400 MHz, DMSO-d ${ }_{6}$ ): $\delta 11.24$ (d, $J=1.9$ $\mathrm{Hz}, 1 \mathrm{H}), 9.77$ (s, 1H), 7.74 (d, $J=7.3 \mathrm{~Hz}, 1 \mathrm{H}), 7.64-7.49$ (m, $3 \mathrm{H}), 7.46-7.29(\mathrm{~m}, 7 \mathrm{H}), 6.89(\mathrm{~d}, J=2.1 \mathrm{~Hz}, 1 \mathrm{H}), 1.23(\mathrm{~s}, 12 \mathrm{H})$. ${ }^{13} \mathrm{C}\left\{{ }^{1} \mathrm{H}\right\}$ NMR $\left(101 \mathrm{MHz}, \mathrm{DMSO}-\mathrm{d}_{6}\right): \delta 168.6,150.7,142.0$, $139.2,132.2$, 132.2, 131.0, 128.5, 128.4, 127.6, 127.6, 124.9, 124.7, 124.1, 123.3, 116.7, 111.3, 83.03, 65.8, 24.8, 24.7.

3-(2-methoxyphenyl)-3-[5-(4,4,5,5-tetramethyl-1,3,2dioxaborolan-2-yl)-3a,7a-dihydro-1H-indol-2-yl]-2,3-dihydro$1 H$-isoindol-1-one (3h). To a $4 \mathrm{~mL}$ vial was added 3-hydroxy3-(2-methoxyphenyl)isoindolin-1-one $(50 \mathrm{mg}, 0.20 \mathrm{mmol})$, $\mathrm{Ca}\left(\mathrm{NTf}_{2}\right)_{2}(1.2 \mathrm{mg}, 0.0020 \mathrm{mmol})$ and $n \mathrm{Bu}_{4} \mathrm{NPF}_{6}(0.8 \mathrm{mg}$, $0.0020 \mathrm{mmol})$ in DCE $(1 \mathrm{~mL})$ at $80^{\circ} \mathrm{C}$. The reaction was stirred for 1 minute and 5-(4,4,5,5-Tetramethyl-1,3,2-dioxaborolan-2yl)-1H-indole ( $71 \mathrm{mg}, 0.29 \mathrm{mmol})$ was added in a single portion and stirred at $80{ }^{\circ} \mathrm{C}$ for 30 mins. Once TLC analysis indicated conversion to the product, the product was concentrated and purified by flash column chromatography $(2: 1$, EtOAc:Hex) to afford an off white solid (92 mg, 98\%). RF (2:1 EtOAc/Hex) = 0.17. IR $v_{\max }\left(\mathrm{cm}^{-1}\right): 3414,3264,3008,1680,1612,1351$. HRMS (ESI) m/z: $[\mathrm{M}+\mathrm{H}]^{+}$Calcd for $\mathrm{C}_{29} \mathrm{H}_{30} \mathrm{BN}_{2} \mathrm{O}_{4} 481.2299$; found 481.2314. ${ }^{1} \mathrm{H}$ NMR (400 MHz, DMSO-d $\left.{ }_{6}\right): \delta 11.06(\mathrm{~d}, J$ $=1.9 \mathrm{~Hz}, 1 \mathrm{H}), 9.16(\mathrm{~s}, 1 \mathrm{H}), 7.79-7.69(\mathrm{~m}, 1 \mathrm{H}), 7.58-7.46$ $(\mathrm{m}, 4 \mathrm{H}), 7.40-7.28(\mathrm{~m}, 3 \mathrm{H}), 7.21(\mathrm{dd}, J=7.7,1.5 \mathrm{~Hz}, 1 \mathrm{H})$, $7.04(\mathrm{~d}, J=7.7 \mathrm{~Hz}, 1 \mathrm{H}), 6.93-6.85(\mathrm{~m}, 1 \mathrm{H}), 6.82(\mathrm{~d}, J=2.4$ $\mathrm{Hz}, 1 \mathrm{H}), 3.51$ (s, 3H), $1.23(\mathrm{~s}, 12 \mathrm{H}) .{ }^{13} \mathrm{C}\left\{{ }^{1} \mathrm{H}\right\} \mathrm{NMR}(101 \mathrm{MHz}$, DMSO-d $\left.{ }_{6}\right): \delta 168.6,157.9,150.7,139.0,131.6,131.4,129.7$, $129.5,128.1,127.7,127.4,127.2,124.7,124.6,123.5,123.1$, 119.9, 117.1, 112.6, 111.1, 83.0, 79.2, 65.4, 55.4, 24.1, 24.7.

3-(furan-2-yl)-3-[5-(4,4,5,5-tetramethyl-1,3,2-dioxaborolan-2yl)-3a,7a-dihydro-1H-indol-2-yl]-2,3-dihydro-1H-isoindol-1one (3i). To a $4 \mathrm{~mL}$ vial was added 3-hydroxy-3-(furan-2yl)isoindolin-1-one (50 mg, $0.23 \mathrm{mmol}), \mathrm{Ca}\left(\mathrm{NTf}_{2}\right)_{2}(1.4 \mathrm{mg}$, $0.0023 \mathrm{mmol})$ and $n \mathrm{Bu}_{4} \mathrm{NPF}_{6}(0.9 \mathrm{mg}, 0.0023 \mathrm{mmol})$ in DCE $(1 \mathrm{~mL})$ at $80^{\circ} \mathrm{C}$. The reaction was stirred for 1 minute and 5- 
(4,4,5,5-Tetramethyl-1,3,2-dioxaborolan-2-yl)-1H-indole $(85 \mathrm{mg}, 0.35 \mathrm{mmol})$ was added in a single portion and stirred at $80{ }^{\circ} \mathrm{C}$ for 30 mins. Once TLC analysis indicated conversion to the product, the product was concentrated and purified by flash column chromatography (1:1 EtOAc:Hex) to afford a pale orange solid $(84 \mathrm{mg}, 82 \%)$. RF $(2: 1 \mathrm{EtOAc} / \mathrm{Hex})=0.35$. IR $\mathrm{v}_{\max }$ ( $\left.\mathrm{cm}^{-1}\right): 3295,3059,2869,1679,1614,1377$. HRMS (ESI) m/z: $[\mathrm{M}+\mathrm{H}]^{+}$Calcd for $\mathrm{C}_{26} \mathrm{H}_{26} \mathrm{BN}_{2} \mathrm{O}_{4} 441.1989$; found 441.1993.. ${ }^{1} \mathrm{H}$ NMR (400 MHz, DMSO-d $\left.)_{6}\right): \delta 11.25(\mathrm{~d}, J=2.1 \mathrm{~Hz}, 1 \mathrm{H}), 9.70$ (s, 1H), $7.80-7.73(\mathrm{~m}, 1 \mathrm{H}), 7.70-7.65(\mathrm{~m}, 1 \mathrm{H}), 7.63-7.50$ $(\mathrm{m}, 3 \mathrm{H}), 7.41-7.30(\mathrm{~m}, 3 \mathrm{H}), 6.97(\mathrm{~d}, J=2.5 \mathrm{~Hz}, 1 \mathrm{H}), 6.45(\mathrm{dd}$, $J=3.2,1.8 \mathrm{~Hz}, 1 \mathrm{H}), 6.30(\mathrm{dd}, J=3.2,0.6 \mathrm{~Hz}, 1 \mathrm{H}), 1.25(\mathrm{~s}$, $12 \mathrm{H}) .{ }^{13} \mathrm{C}\left\{{ }^{1} \mathrm{H}\right\}$ NMR (101 MHz, DMSO-d 6 ): $\delta$ 169.0, 155.3, $149.5,143.7,139.4,132.6,131.6,129.2$, 127.9, 127.5, 125.0, $124.4,123.6,115.5,111.8,110.9,107.5,83.5,62.6,25.3,25.2$.

\section{3-[3,5-bis(trifluoromethyl)phenyl]-3-[5-(4,4,5,5-tetramethyl-} 1,3,2-dioxaborolan-2-yl)-3a,7a-dihydro-1H-indol-2-yl]-2,3dihydro-1H-isoindol-1-one (3j). To a $4 \mathrm{~mL}$ vial was added 3hydroxy-3-(3,5-bis(trifluoromethyl))isoindolin-1-one $(50 \mathrm{mg}$, $0.14 \mathrm{mmol}), \mathrm{Ca}\left(\mathrm{NTf}_{2}\right)_{2}(0.8 \mathrm{mg}, 0.0014 \mathrm{mmol})$ and $n \mathrm{Bu}_{4} \mathrm{NPF}_{6}$ $(0.5 \mathrm{mg}, 0.0014 \mathrm{mmol})$ in $\mathrm{DCE}(1 \mathrm{~mL})$ at $80^{\circ} \mathrm{C}$. The reaction was stirred for 1 minute and 5-(4,4,5,5-Tetramethyl-1,3,2dioxaborolan-2-yl)-1H-indole (51 $\mathrm{mg}, 0.21 \mathrm{mmol})$ was added in a single portion and stirred at $80{ }^{\circ} \mathrm{C}$ for 30 mins. Once TLC analysis indicated conversion to the product, the product was concentrated and purified by flash column chromatography $(1: 1$, EtOAc:Hex) to afford an off white solid $(66 \mathrm{mg}, 81 \%)$. RF $(1: 1 \mathrm{EtOAc} / \mathrm{Hex})=0.30$. IR $\mathrm{v}_{\max }\left(\mathrm{cm}^{-1}\right): 3273,2980,1695$, 1355, 1275, 1131. HRMS (ESI) $\mathrm{m} / \mathrm{z}:[\mathrm{M}+\mathrm{H}]^{+}$Calcd for $\mathrm{C}_{30} \mathrm{H}_{26} \mathrm{BF}_{6} \mathrm{~N}_{2} \mathrm{O}_{3}$ 587.1941; found 587.1947.. ${ }^{1} \mathrm{H}$ NMR (400 $\left.\mathrm{MHz} \mathrm{CDCl}_{3}\right): \delta 8.56(\mathrm{~s}, 1 \mathrm{H}), 7.99-7.88(\mathrm{~m}, 3 \mathrm{H}), 7.81(\mathrm{~s}, 1 \mathrm{H})$, $7.69-7.57(\mathrm{~m}, 2 \mathrm{H}), 7.57-7.48(\mathrm{~m}, 1 \mathrm{H}), 7.43(\mathrm{~d}, J=7.6 \mathrm{~Hz}$, $1 \mathrm{H}), 7.41-7.29(\mathrm{~m}, 2 \mathrm{H}), 7.18(\mathrm{~s}, 1 \mathrm{H}), 1.28(\mathrm{~s}, 12 \mathrm{H}) .{ }^{13} \mathrm{C}\left\{{ }^{1} \mathrm{H}\right\}$ NMR $\left(101 \mathrm{MHz}, \mathrm{CDCl}_{3}\right): \delta 170.2,149.6,145.3,139.4,133.2$ $\left(\mathrm{q}, J_{F}=33.4 \mathrm{~Hz}\right), 132.0,130.1,129.3,129.3,126.8,126.8$, $125.0,124.7,124.6,123.8,123.6,122.2,121.9,116.3,111.6$, $83.75,66.24,24.9,24.8$.

3-phenyl-3-phenylsulfanyl-2,3-dihydro-isoindol-1-one (4a). To a $4 \mathrm{~mL}$ vial was added 3-hydroxy-3-phenylisoindolin-1-one (50 $\mathrm{mg}, 0.22 \mathrm{mmol}), \mathrm{Ca}\left(\mathrm{NTf}_{2}\right)_{2}(3 \mathrm{mg}, 0.0022 \mathrm{mmol})$ and $n \mathrm{Bu}_{4} \mathrm{NPF}_{6}(1.7 \mathrm{mg}, 0.0022 \mathrm{mmol})$ in DCE $(1 \mathrm{~mL})$ at $80^{\circ} \mathrm{C}$. The reaction was stirred for 5 minutes and thiophenol $(29 \mathrm{mg}, 0.27$ mmol) was added in a single portion and stirred at $80{ }^{\circ} \mathrm{C}$ for 15 minutes. Once TLC analysis indicated conversion to the product, the product was concentrated and purified by flash column chromatography $(1: 1$, EtOAc:Hex $)$ to afford a white solid $(54 \mathrm{mg}, 77 \%)$. RF $(1: 1 \mathrm{EtOAc} / \mathrm{Hex})=0.43$. IR $\mathrm{v}_{\max }\left(\mathrm{cm}^{-}\right.$ $\left.{ }^{1}\right)$ : 3062, 2848, 1696, 1494, 1345, 742. HRMS (ESI) m/z: $[\mathrm{M}+\mathrm{H}]^{+}$Calcd for $\mathrm{C}_{20} \mathrm{H}_{16} \mathrm{NOS}$ 318.0953; found 318.0947. ${ }^{1} \mathrm{H}$ NMR $\left(400 \mathrm{MHz}, \mathrm{CDCl}_{3}\right): \delta 7.84-7.75(\mathrm{~m}, 2 \mathrm{H}), 7.73-7.67$ $(\mathrm{m}, 1 \mathrm{H}), 7.56(\mathrm{td}, J=7.5,1.1 \mathrm{~Hz}, 1 \mathrm{H}), 7.51-7.29(\mathrm{~m}, 6 \mathrm{H}), 7.24$ $-7.18(\mathrm{~m}, 1 \mathrm{H}), 7.14(\mathrm{dt}, J=8.2,1.7 \mathrm{~Hz}, 1 \mathrm{H}), 7.08-7.02(\mathrm{~m}$, 2H). ${ }^{13} \mathrm{C}\left\{{ }^{1} \mathrm{H}\right\}$ NMR (101 MHz, $\left.\mathrm{CDCl}_{3}\right): \delta 169.2,148.7,139.1$, $137.1,132.6,129.9$, 129.8, 129.4, 129.2, 129.0, 128.7, 128.6, $126.1,123.9,123.4,75.79$.

Reaction also performed on a $2.0 \mathrm{mmol}$ from 3-hydroxy-3phenylisoindolin-1-one (450 mg, $2.0 \mathrm{mmol}), \mathrm{Ca}\left(\mathrm{NTf}_{2}\right)_{2}(12 \mathrm{mg}$, $0.02 \mathrm{mmol})$ and $n \mathrm{Bu}_{4} \mathrm{NPF}_{6}(8 \mathrm{mg}, 0.02 \mathrm{mmol})$ with thiophenol $(265 \mathrm{mg}, 2.40 \mathrm{mmol})$ in DCE $(9 \mathrm{~mL})$ for $30 \mathrm{~min}$. Purification $(1: 1 \mathrm{EtOAc:Hex})$ afforded the product as a white solid $(608 \mathrm{mg}$, $96 \%)$ 3-(4-methoxyphenyl)-3-(phenylthio)isoindolin-1-one (4b). To a $4 \mathrm{~mL}$ vial was added 3-(4-methoxyphenyl)-3hydroxyisoindolin-1-one (60 mg, $0.24 \mathrm{mmol}), \mathrm{Ca}\left(\mathrm{NTf}_{2}\right)_{2}(1.5$ $\mathrm{mg}, 0.0024 \mathrm{mmol})$ and $n \mathrm{Bu}_{4} \mathrm{NPF}_{6}(1 \mathrm{mg}, 0.0024 \mathrm{mmol})$ in DCE $(1 \mathrm{~mL})$ at $80{ }^{\circ} \mathrm{C}$. The reaction was stirred for 5 minutes and thiophenol (39 $\mathrm{mg}, 0.353 \mathrm{mmol}$ ) was added in a single portion and stirred at $80{ }^{\circ} \mathrm{C}$ for 15 minutes. Once TLC analysis indicated conversion to the product, the product was concentrated and purified by flash column chromatography $(1: 1 \mathrm{EtOAc:Hex})$ to afford a white solid $(70 \mathrm{mg}, 86 \%)$. RF $(1: 1 \mathrm{EtOAc} / \mathrm{CycHex})=$ 0.53. IR $v_{\max }\left(\mathrm{cm}^{-1}\right): 3057,2930,2849,1700,1606,1509$. HRMS (ESI) m/z: $[\mathrm{M}+\mathrm{H}]^{+}$Calcd for $\mathrm{C}_{21} \mathrm{H}_{18} \mathrm{NO}_{2} \mathrm{~S} 348.1058$; found 348.1053. ${ }^{1} \mathrm{H}$ NMR $\left(400 \mathrm{MHz}, \mathrm{CDCl}_{3}\right): \delta 7.74-7.64(\mathrm{~m}$, $3 \mathrm{H}), 7.61-7.51(\mathrm{~m}, 1 \mathrm{H}), 7.46(\mathrm{~d}, J=7.5 \mathrm{~Hz}, 1 \mathrm{H}), 7.35-7.28$ (m, 1H), $7.24-7.18(\mathrm{~m}, 1 \mathrm{H}), 7.17-7.11(\mathrm{~m}, 3 \mathrm{H}), 7.11-7.01$ (m, 2H), $6.97-6.88(\mathrm{~m}, 2 \mathrm{H}), 3.81(\mathrm{~s}, 3 \mathrm{H}) .{ }^{13} \mathrm{C}\left\{{ }^{1} \mathrm{H}\right\}$ NMR $(101$ $\left.\mathrm{MHz}, \mathrm{CDCl}_{3}\right)$ : 169.1, 160.0, 148.9, 137.1, 132.5, 131.1, 131.04, $129.9,129.8,129.6,128.6,127.5,123.8,123.4,114.4,75.5$, 55.5.

3-(4-chlorophenyl)-3-(phenylthio)isoindolin-1-one (4c). To a 4 $\mathrm{mL}$ vial was added 3-(4-chlorophenyl)-3-hydroxyisoindolin-1one (100 mg, $0.39 \mathrm{mmol}), \mathrm{Ca}\left(\mathrm{NTf}_{2}\right)_{2}(2 \mathrm{mg}, 0.0039 \mathrm{mmol})$ and $n \mathrm{Bu}_{4} \mathrm{NPF}_{6}(1.5 \mathrm{mg}, 0.0039 \mathrm{mmol})$ in DCE $(2 \mathrm{~mL})$ at $80^{\circ} \mathrm{C}$. The reaction was stirred for 5 minutes and thiophenol $(64 \mathrm{mg}, 0.578$ mmol) was added in a single portion and stirred at $80^{\circ} \mathrm{C}$ for 15 minutes. Once TLC analysis indicated conversion to the product, the product was concentrated and purified by flash column chromatography $(1: 1$, EtOAC:Hex $)$ to afford a white solid $(116 \mathrm{mg}, 86 \%)$ RF $(1: 1 \mathrm{EtOAc} / \mathrm{CycHex})=0.6$. IR $v_{\max }$ $\left(\mathrm{cm}^{-1}\right): 3130,3062,2851,1702,1470,1096$. HRMS (ESI) m/z: $[\mathrm{M}+\mathrm{H}]^{+}$Calcd for $\mathrm{C}_{20} \mathrm{H}_{15} \mathrm{CINOS} 352.0563$; found $352.0559 .{ }^{1} \mathrm{H}$ NMR (400 MHz, DMSO-d $): 9.84(\mathrm{~s}, 1 \mathrm{H}), 7.98-7.72(\mathrm{~m}, 3 \mathrm{H})$, $7.72-7.59(\mathrm{~m}, 1 \mathrm{H}), 7.58-7.56(\mathrm{~m}, 2 \mathrm{H}), 7.45-7.20(\mathrm{~m}, 3 \mathrm{H})$, $7.20-6.90(\mathrm{~m}, 4 \mathrm{H}) .{ }^{13} \mathrm{C}\left\{{ }^{1} \mathrm{H}\right\}$ NMR (101 MHz, DMSO-d 6 ): $\delta 167.7,147.5,138.5,136.71,133.4,132.4,130.3,129.7,129.1$, $128.9,128.8,128.4,128.0,124.0,122.4,74.9$.

4-(1-Hydroxy-3-oxoisoindolin-1-yl)benzonitrile (4d). To a 4 $\mathrm{mL}$ vial was added 4-(1-hydroxy-3-oxoisoindolin-1yl)benzonitrile $(50 \mathrm{mg}, 0.20 \mathrm{mmol}), \mathrm{Ca}\left(\mathrm{NTf}_{2}\right)_{2}(1.2 \mathrm{mg}, 0.002$ $\mathrm{mmol})$ and $n \mathrm{Bu}_{4} \mathrm{NPF}_{6}(0.8 \mathrm{mg}, 0.002 \mathrm{mmol})$ in DCE $(1 \mathrm{~mL})$ at $80{ }^{\circ} \mathrm{C}$. The reaction was stirred for 5 minutes and thiophenol $(33$ $\mathrm{mg}, 0.3 \mathrm{mmol}$ ) was added in a single portion and stirred at 80 ${ }^{\circ} \mathrm{C}$ for 15 minutes. Once TLC analysis indicated conversion to the product, the product was concentrated and purified by flash column chromatography $(1: 1$, EtOAc:Hex $)$ to afford a cream solid (54 mg, 79\%). RF (1:1 EtOAc/CycHex $)=0.50$. IR $v_{\max }$ $\left(\mathrm{cm}^{-1}\right): 3215,3075,2229,1714,1679,1497 . \mathrm{HRMS}$ (ESI) m/z: $[\mathrm{M}+\mathrm{H}]^{+}$Calcd for $\mathrm{C}_{21} \mathrm{H}_{15} \mathrm{~N}_{2} \mathrm{OS}$ 343.0905; found 343.0907. ${ }^{1} \mathrm{H}$ NMR (400 MHz, $\left.\mathrm{CDCl}_{3}\right): \delta 7.93(\mathrm{~d}, J=8.5 \mathrm{~Hz}, 2 \mathrm{H}), 7.71(\mathrm{~d}, J$ $=8.5 \mathrm{~Hz}, 2 \mathrm{H}), 7.68-7.57(\mathrm{~m}, 2 \mathrm{H}), 7.54-7.46(\mathrm{~m}, 2 \mathrm{H}), 7.38$ $(\mathrm{t}, J=7.3 \mathrm{~Hz}, 1 \mathrm{H}), 7.25-7.21(\mathrm{~m}, 1 \mathrm{H}), 7.14(\mathrm{~d}, J=6.9 \mathrm{~Hz}$, 2H), $7.11-7.04(\mathrm{~m}, 2 \mathrm{H}) .{ }^{13} \mathrm{C}\left\{{ }^{1} \mathrm{H}\right\}$ NMR $\left(101 \mathrm{MHz}, \mathrm{CDCl}_{3}\right): \delta$ $169.3,147.7,144.5,137.1,133.0,132.9,130.2,129.3,128.9$, $128.7,127.2,123.8,123.7,118.4,112.9,75.1$.

3-(4-trifluoromethylphenyl)-3-(phenylthio)isoindolin-1-one (4e). To a $4 \mathrm{~mL}$ vial was added 3-hydroxy-3-(4(trifluoromethyl)phenyl)isoindolin-1-one (60 mg, $0.21 \mathrm{mmol})$, $\mathrm{Ca}\left(\mathrm{NTf}_{2}\right)_{2}(1.2 \mathrm{mg}, 0.0021 \mathrm{mmol})$ and $n \mathrm{Bu}_{4} \mathrm{NPF}_{6}(0.8 \mathrm{mg}$, 
$0.0021 \mathrm{mmol})$ in DCE $(1 \mathrm{~mL})$ at $80^{\circ} \mathrm{C}$. The reaction was stirred for 5 minutes and thiophenol ( $34 \mathrm{mg}, 0.307 \mathrm{mmol}$ ) was added in a single portion and stirred at $80{ }^{\circ} \mathrm{C}$ for 15 minutes. Once TLC analysis indicated conversion to the product, the product was concentrated and purified by flash column chromatography (1:1, EtOAc:Hex) to afford a white solid (76 mg, 96\%). RF (1:1 EtOAc/CycHex $)=0.60 . I_{\text {IR }} v_{\max }\left(\mathrm{cm}^{-1}\right): 3126,3071,1699,1496$, 1392. HRMS (ESI) $\mathrm{m} / \mathrm{z}$ : $[\mathrm{M}+\mathrm{H}]^{+}$Calcd for $\mathrm{C}_{21} \mathrm{H}_{15} \mathrm{~F}_{3} \mathrm{NOS}$ 386.0826; found 386.0823.. ${ }^{1} \mathrm{H} \mathrm{NMR}\left(400 \mathrm{MHz}, \mathrm{CDCl}_{3}\right): \delta 7.93$ $(\mathrm{d}, J=8.3 \mathrm{~Hz}, 1 \mathrm{H}), 7.73-7.62(\mathrm{~m}, 2 \mathrm{H}), 7.59$ (t, $J=7.0 \mathrm{~Hz}$, $1 \mathrm{H}), 7.50(\mathrm{~d}, J=7.5 \mathrm{~Hz}, 1 \mathrm{H}), 7.36(\mathrm{t}, J=7.3 \mathrm{~Hz}, 1 \mathrm{H}), 7.24$ $7.23(\mathrm{~m}, 3.6 \mathrm{~Hz}, 1 \mathrm{H}), 7.15-7.07(\mathrm{~m}, 3 \mathrm{H}) .{ }^{13} \mathrm{C}\left\{{ }^{1} \mathrm{H}\right\}$ NMR $(101$ $\left.\mathrm{MHz}, \mathrm{CDCl}_{3}\right): \delta 169.1,148.1,143.3,137.1,132.9,130.1,129.7$, $129.2,128.9,128.8,126.7,126.2,126.2,123.7,75.2$.

3-(2-methoxyphenyl)-3-(phenylthio)isoindolin-1-one (4f). To a $4 \mathrm{~mL}$ vial was added 3-(2-methoxyphenyl)-3hydroxyisoindolin-1-one (100 mg, $0.39 \mathrm{mmol}), \mathrm{Ca}\left(\mathrm{NTf}_{2}\right)_{2}(2.4$ $\mathrm{mg}, 0.0039 \mathrm{mmol})$ and $n \mathrm{Bu}_{4} \mathrm{NPF}_{6}(1.5 \mathrm{mg}, 0.0039 \mathrm{mmol})$ in DCE $(2 \mathrm{~mL})$ at $80{ }^{\circ} \mathrm{C}$. The reaction was stirred for 5 minutes and thiophenol $(65 \mathrm{mg}, 0.353 \mathrm{mmol})$ was added in a single portion and stirred at $80{ }^{\circ} \mathrm{C}$ for 15 minutes. Once TLC analysis indicated conversion to the product, the product was concentrated and purified by flash column chromatography (1:1, EtOAc:Hex) to afford a white solid (115 mg, 84\%). RF $(1: 1 \mathrm{EtOAc} / \mathrm{CycHex})=0.40$. IR $v_{\max }\left(\mathrm{cm}^{-1}\right): 3283,3051,2932$, 1692, 1489, 1249. HRMS (ESI) $\mathrm{m} / \mathrm{z}:[\mathrm{M}+\mathrm{H}]^{+}$Calcd for $\mathrm{C}_{21} \mathrm{H}_{18} \mathrm{NO}_{2} \mathrm{~S}$ 348.1058; found 348.1050. ${ }^{1} \mathrm{H}$ NMR (400 MHz, $\left.\mathrm{CDCl}_{3}\right): \delta 7.95-7.80(\mathrm{~m}, 2 \mathrm{H}), 7.64(\mathrm{td}, J=7.6,1.1 \mathrm{~Hz}, 1 \mathrm{H})$, $7.59-7.47(\mathrm{~m}, 2 \mathrm{H}), 7.44-7.32(\mathrm{~m}, 2 \mathrm{H}), 7.21-7.14(\mathrm{~m}, 1 \mathrm{H})$, $7.14-7.09$ (m, 2H), $7.08-7.01(\mathrm{~m}, 3 \mathrm{H}), 6.92(\mathrm{td}, J=7.7,1.1$ $\mathrm{Hz}, 1 \mathrm{H}), 4.02(\mathrm{~s}, 3 \mathrm{H}) .{ }^{13} \mathrm{C}\left\{{ }^{1} \mathrm{H}\right\} \mathrm{NMR}\left(101 \mathrm{MHz}, \mathrm{CDCl}_{3}\right): \delta$ $168.0,157.8,146.4,136.8,131.5,131.4,130.5,129.4,128.7$, $128.4,127.4,126.5,125.6,123.5,120.8,112.8,74.5,56.2$.

\section{3-(3,5-bis(trifluoromethyl))-3-(phenylthio)isoindolin-1-one}

(4g). To a $4 \mathrm{~mL}$ vial was added 3-hydroxy-3-(3,5bis(trifluoromethyl))isoindolin-1-one (100 mg, $0.28 \mathrm{mmol})$, $\mathrm{Ca}\left(\mathrm{NTf}_{2}\right)_{2}(1.7 \mathrm{mg}, 0.0028 \mathrm{mmol})$ and $n \mathrm{Bu}_{4} \mathrm{NPF}_{6}(1 \mathrm{mg}, 0.0028$ $\mathrm{mmol})$ in DCE $(2 \mathrm{~mL})$ at $80^{\circ} \mathrm{C}$. The reaction was stirred for 5 minutes and thiophenol (46 $\mathrm{mg}, 0.42 \mathrm{mmol}$ ) was added in a single portion and stirred at $80{ }^{\circ} \mathrm{C}$ for 15 minutes. Once TLC analysis indicated conversion to the product, the product was concentrated and purified by flash column chromatography (2:3, EtOAc:Hex) to afford a white solid (113 mg, 90\%). RF $(2: 3 \mathrm{EtOAc} / \mathrm{CycHex})=0.56 . \mathrm{IR} \mathrm{v}_{\max }\left(\mathrm{cm}^{-1}\right): 3208,3094,1715$, 1469, 1312, 1280. HRMS (ESI) $\mathrm{m} / \mathrm{z}:[\mathrm{M}+\mathrm{H}]^{+}$Calcd for $\mathrm{C}_{22} \mathrm{H}_{14} \mathrm{~F}_{6} \mathrm{NOS}$ 454.0700; found 454.0699. ${ }^{1} \mathrm{H}$ NMR (400 MHz, $\left.\mathrm{CDCl}_{3}\right): \delta 8.26(\mathrm{~s}, 2 \mathrm{H}), 7.89(\mathrm{~s}, 1 \mathrm{H}), 7.64-7.62(\mathrm{~m}, 2 \mathrm{H}), 7.53$ $(\mathrm{d}, J=7.7 \mathrm{~Hz}, 1 \mathrm{H}), 7.47-7.36(\mathrm{~m}, 1 \mathrm{H}), 7.28-7.24(\mathrm{~m}, 2 \mathrm{H})$, $7.20-7.05(\mathrm{~m}, 4 \mathrm{H}) .{ }^{13} \mathrm{C}\left\{{ }^{1} \mathrm{H}\right\} \mathrm{NMR}\left(101 \mathrm{MHz}, \mathrm{CDCl}_{3}\right): \delta 167.6$, $146.1,142.6,136.7,131.6(\mathrm{~d}, J=238 \mathrm{~Hz}), 131.1,130.8$, , $130.1,129.4,128.6,128.6,126.8,124.2,123.1(\mathrm{~d}, J=275 \mathrm{~Hz})$, $123.0,122.8,, 74.5$.

3-(thiophen-2-yl)-3-(phenylthio)isoindolin-1-one (4h). To a 4 $\mathrm{mL}$ vial was added 3-hydroxy-3-(thiophen-2-yl)isoindolin-1one (100 mg, $0.43 \mathrm{mM}), \mathrm{Ca}\left(\mathrm{NTf}_{2}\right)_{2}(2.4 \mathrm{mg}, 0.0043 \mathrm{mmol})$ and $n \mathrm{Bu}_{4} \mathrm{NPF}_{6}(1.5 \mathrm{mg}, 0.0043 \mathrm{mmol})$ in DCE $(2 \mathrm{~mL})$ at $80^{\circ} \mathrm{C}$. The reaction was stirred for 5 minutes and thiophenol $(72 \mathrm{mg}, 0.65$ mmol) was added in a single portion and stirred at $80^{\circ} \mathrm{C}$ for 15 minutes. Once TLC analysis indicated conversion to the product, the product was concentrated and purified by flash column chromatography (1:1, EtOAc:Hex) to afford a light brown solid $(120 \mathrm{mg}, 86 \%)$. RF $(1: 1 \mathrm{EtOAc} / \mathrm{CycHex})=0.60$. IR $v_{\max }\left(\mathrm{cm}^{-1}\right): 3155,3060,2836,1691,1470,1354$. HRMS (ESI) $\mathrm{m} / \mathrm{z}$ : $[\mathrm{M}+\mathrm{H}]^{+}$Calcd for $\mathrm{C}_{18} \mathrm{H}_{14} \mathrm{NOS}_{2}$ 324.0517; found 324.0516. ${ }^{1} \mathrm{H}$ NMR $\left(400 \mathrm{MHz}, \mathrm{CDCl}_{3}\right): \delta 7.75(\mathrm{~d}, J=7.8 \mathrm{~Hz}$, $1 \mathrm{H}), 7.59(\mathrm{td}, J=7.5,0.9 \mathrm{~Hz}, 1 \mathrm{H}), 7.50(\mathrm{~d}, J=7.5 \mathrm{~Hz}, 1 \mathrm{H})$, $7.39-7.29(\mathrm{~m}, 3 \mathrm{H}), 7.24-7.23(\mathrm{~m}, 1 \mathrm{H}), 7.18-7.16(\mathrm{~m}, 2 \mathrm{H})$, $7.13-7.05(\mathrm{~m}, 2 \mathrm{H}), 7.01(\mathrm{dd}, J=5.0,3.7 \mathrm{~Hz}, 1 \mathrm{H}), 6.92-6.91$ $(\mathrm{m}, 1 \mathrm{H}) .{ }^{13} \mathrm{C}\left\{{ }^{1} \mathrm{H}\right\}$ NMR $\left(101 \mathrm{MHz}, \mathrm{CDCl}_{3}\right): \delta 168.8,148.2$, $143.0,136.9,132.7,130.0,129.5,129.5,129.1,128.7,127.6$, $126.4,125.7,123.8,123.4,72.8$.

3-(furan-2-yl)-3-(phenylthio)isoindolin-1-one (4i). To a $4 \mathrm{~mL}$ vial was added 3-hydroxy-3-(furan-2-yl)isoindolin-1-one (50 $\mathrm{mg}, 0.23 \mathrm{mmol}), \mathrm{Ca}\left(\mathrm{NTf}_{2}\right)_{2}(1.4 \mathrm{mg}, 0.0023 \mathrm{mmol})$ and $n \mathrm{Bu}_{4} \mathrm{NPF}_{6}(1 \mathrm{mg}, 0.0023 \mathrm{mmol})$ in DCE $(1 \mathrm{~mL})$ at $80{ }^{\circ} \mathrm{C}$. The reaction was stirred for 5 minutes and thiophenol $(38 \mathrm{mg}, 0.35$ mmol) was added in a single portion and stirred at $80^{\circ} \mathrm{C}$ for 15 minutes. Once TLC analysis indicated conversion to the product, the product was concentrated and purified by flash column chromatography $(1: 1$, EtOAc:Hex $)$ to afford a light brown solid (70 mg, 98\%). RF (1:1 EtOAc/CycHex) =0.6. IR $\mathrm{v}_{\max }\left(\mathrm{cm}^{-1}\right): 3153,3060,1689,1500,1307,1154$. HRMS (ESI) $\mathrm{m} / \mathrm{z}$ : $[\mathrm{M}+\mathrm{H}]^{+}$Calcd for $\mathrm{C}_{18} \mathrm{H}_{14} \mathrm{NO}_{2} \mathrm{~S} 308.0745$; found 308.0748 . ${ }^{1} \mathrm{H}$ NMR $\left(400 \mathrm{MHz}, \mathrm{CDCl}_{3}\right): \delta 7.86(\mathrm{t}, J=10.3 \mathrm{~Hz}, 1 \mathrm{H}), 7.65$ $-7.57(\mathrm{~m}, 2 \mathrm{H}), 7.51(\mathrm{~d}, J=0.9 \mathrm{~Hz}, 1 \mathrm{H}), 7.42(\mathrm{t}, J=7.6 \mathrm{~Hz}$, 1H), $7.31-7.20(\mathrm{~m}, 2 \mathrm{H}), 7.18-7.10(\mathrm{~m}, 4 \mathrm{H}), 6.94(\mathrm{~s}, 1 \mathrm{H}), 6.37$ $-6.28(\mathrm{~m}, 2 \mathrm{H}) .{ }^{13} \mathrm{C}\left\{{ }^{1} \mathrm{H}\right\}$ NMR (101 MHz, DMSOd- 6 ): $\delta 167.6$, $150.7,145.8,144.0,136.7,132.4,130.4,129.7,129.2$, 128.6, $128.5,124.1,122.4,110.9,108.2,70.6$.

3-(benzo[d][1,3]dioxol-5-yl)-3-(phenylthio)isoindolin-1-one $(4 j)$. To a $4 \mathrm{~mL}$ vial was added 3-(benzo[d][1,3]dioxol-5yl)isoindolin-1-one $(80 \mathrm{mg}, 0.30 \mathrm{mmol}), \mathrm{Ca}\left(\mathrm{NTf}_{2}\right)_{2}(1.8 \mathrm{mg}$, $0.003 \mathrm{mmol})$ and $n \mathrm{Bu}_{4} \mathrm{NPF}_{6}(1.2 \mathrm{mg}, 0.003 \mathrm{mmol})$ in DCE (1 $\mathrm{mL})$ at $80{ }^{\circ} \mathrm{C}$. The reaction was stirred for 5 minutes and thiophenol (33 mg, $0.3 \mathrm{mmol}$ ) was added in a single portion and stirred at $80{ }^{\circ} \mathrm{C}$ for 15 minutes. Once TLC analysis indicated conversion to the product, the product was concentrated and purified by flash column chromatography $(1: 1$, EtOAc:Hex $)$ to afford a peach solid $(98 \mathrm{mg}, 91 \%)$. RF $(1: 1 \mathrm{EtOAc} / \mathrm{CycHex})=$ 0.56. IR $v_{\max }\left(\mathrm{cm}^{-1}\right): 3209,2902,1706,1671,1485,1246$. HRMS (ESI) m/z: $[\mathrm{M}+\mathrm{H}]^{+}$Calcd for $\mathrm{C}_{21} \mathrm{H}_{16} \mathrm{NO}_{3} \mathrm{~S} 362.0851$; found 362.0852. ${ }^{1} \mathrm{H}$ NMR (400 MHz, $\left.\mathrm{CDCl}_{3}\right)$ : $\delta 9.74(\mathrm{~s}, 1 \mathrm{H})$, $7.88(\mathrm{~d}, J=7.7 \mathrm{~Hz}, 1 \mathrm{H}), 7.62(\mathrm{t}, J=7.4 \mathrm{~Hz}, 1 \mathrm{H}), 7.38-7.30$ $(\mathrm{m}, 2 \mathrm{H}), 7.29-7.18(\mathrm{~m}, 4 \mathrm{H}), 7.10(\mathrm{~d}, J=4.3 \mathrm{~Hz}, 5 \mathrm{H}), 6.96(\mathrm{~d}$, $J=8.2 \mathrm{~Hz}, 1 \mathrm{H}), 6.06(\mathrm{~s}, 2 \mathrm{H}) .{ }^{13} \mathrm{C}\left\{{ }^{1} \mathrm{H}\right\} \mathrm{NMR}(101 \mathrm{MHz}$, $\left.\mathrm{CDCl}_{3}\right): \delta 167.6,147.7,147.4,136.6,133.2,132.1,130.4,129.7$, $129.5,128.6,128.3,124.2,122.3,119.3,108.2,106.8,101.6$, 75.30 .

3-((4-methoxyphenyl)thio)-3-phenylisoindolin-1-one (6a). To a $4 \mathrm{~mL}$ vial was added 3-hydroxy-3-phenylisoindolin-1-one (100 $\mathrm{mg}, 0.44 \mathrm{mmol}), \mathrm{Ca}\left(\mathrm{NTf}_{2}\right)_{2}$ (3 $\left.\mathrm{mg}, 0.0044 \mathrm{mmol}\right)$ and $n \mathrm{Bu}_{4} \mathrm{NPF}_{6}(1.7 \mathrm{mg}, 0.0044 \mathrm{mmol})$ in DCE $(2 \mathrm{~mL})$ at $80^{\circ} \mathrm{C}$. The reaction was stirred for 5 minutes and 4-methoxythiophenol (75 $\mathrm{mg}, 0.533 \mathrm{mmol}$ ) was added in a single portion and stirred at 80 ${ }^{\circ} \mathrm{C}$ for 15 minutes. Once TLC analysis indicated conversion to the product, the product was concentrated and purified by flash column chromatography $(1: 1$, EtOAc:Hex $)$ to afford a cream solid $(121 \mathrm{mg}, 78 \%)$. RF $(1: 1 \mathrm{EtOAc} / \mathrm{Hex})=0.26$. IR $\mathrm{v}_{\max }(\mathrm{cm}$ 
1): 3198, 3078, 2840, 1698, 1589, 1243. HRMS (ESI) m/z: $[\mathrm{M}+\mathrm{H}]^{+}$Calcd for $\mathrm{C}_{21} \mathrm{H}_{18} \mathrm{NO}_{2} \mathrm{~S} 348.1058$; found $348.1059 .{ }^{1} \mathrm{H}$ NMR $\left(400 \mathrm{MHz}, \mathrm{DMSO}_{6}\right): \delta 9.73(\mathrm{~s}, 1 \mathrm{H}), 7.86(\mathrm{~d}, J=7.8 \mathrm{~Hz}$, $1 \mathrm{H}), 7.78(\mathrm{~d}, J=7.6 \mathrm{~Hz}, 2 \mathrm{H}), 7.62(\mathrm{t}, J=7.5 \mathrm{~Hz}, 1 \mathrm{H}), 7.46(\mathrm{t}$, $J=7.5 \mathrm{~Hz}, 2 \mathrm{H}), 7.41-7.23(\mathrm{~m}, 3 \mathrm{H}), 7.02(\mathrm{~d}, J=8.7 \mathrm{~Hz}, 2 \mathrm{H})$, $6.67(\mathrm{~d}, J=8.7 \mathrm{~Hz}, 2 \mathrm{H}), 3.66(\mathrm{~s}, 3 \mathrm{H}) .{ }^{13} \mathrm{C}\left\{{ }^{1} \mathrm{H}\right\} \mathrm{NMR}(101 \mathrm{MHz}$, DMSO-d $\left.{ }_{6}\right): \delta 167.8,160.4,148.0,139.5,138.4,132.1,130.5$, $128.8,128.6,128.5,126.0,124.0,122.3,120.1,113.9,75.4$, 55.2 .

\section{3-((4-(trifluoromethyl)phenyl)thio)-3-phenylisoindolin-1-one}

$(\boldsymbol{6 b})$. To a $4 \mathrm{~mL}$ vial was added 3-hydroxy-3-phenylisoindolin1-one (100 mg, $0.44 \mathrm{mmol}), \mathrm{Ca}\left(\mathrm{NTf}_{2}\right)_{2}(2.7 \mathrm{mg}, 0.0044 \mathrm{mmol})$ and $n \mathrm{Bu}_{4} \mathrm{NPF}_{6}(1.7 \mathrm{mg}, 0.0044 \mathrm{mmol})$ in DCE $(2 \mathrm{~mL})$ at $80^{\circ} \mathrm{C}$. The reaction was stirred for 5 minutes and 4(trifluoromethyl)thiophenol (95 mg, $0.533 \mathrm{mmol}$ ) was added in a single portion and stirred at $80^{\circ} \mathrm{C}$ for 15 minutes. Once TLC analysis indicated conversion to the product, the product was concentrated and purified by flash column chromatography (1:1, EtOAc:Hex) to afford a cream solid (142 mg, 83\%). RF $(1: 1 \mathrm{EtOAc} / \mathrm{Hex})=0.54$. IR $v_{\max }\left(\mathrm{cm}^{-1}\right): 3160,3062,2850$, 1694, 1319, 1124. HRMS (ESI) $\mathrm{m} / \mathrm{z}:[\mathrm{M}+\mathrm{H}]^{+}$Calcd for $\mathrm{C}_{21} \mathrm{H}_{15} \mathrm{~F}_{3} \mathrm{NOS} 386.0826$; found 386.0828. ${ }^{1} \mathrm{H}$ NMR (400 MHz, DMSO-d $\left.{ }_{6}\right): \delta 9.96(\mathrm{~s}, 1 \mathrm{H}), 7.90(\mathrm{~d}, J=7.8 \mathrm{~Hz}, 1 \mathrm{H}), 7.80(\mathrm{~d}, J$ $=7.5 \mathrm{~Hz}, 2 \mathrm{H}), 7.65(\mathrm{t}, J=7.2 \mathrm{~Hz}, 1 \mathrm{H}), 7.54-7.45(\mathrm{~m}, 4 \mathrm{H})$, $7.45-7.35(\mathrm{~m}, 2 \mathrm{H}), 7.35-7.27(\mathrm{~m}, 3 \mathrm{H}) .{ }^{13} \mathrm{C}\left\{{ }^{1} \mathrm{H}\right\}$ NMR $(101$ MHz, DMSO-d $\left.\mathrm{d}_{6}\right): \delta 167.7,147.5,139.1,137.0,134.9,132.5$, $130.2,129.8,129.4,129.0,128.8,126.0,125.1,125.1,124.1$, 122.4, 75.8 .

3-((4-chlorophenyl)thio)-3-phenylisoindolin-1-one (6c). To a 4 $\mathrm{mL}$ vial was added 3-hydroxy-3-phenylisoindolin-1-one (100 $\mathrm{mg}, 0.44 \mathrm{mmol}), \mathrm{Ca}\left(\mathrm{NTf}_{2}\right)_{2}(2.67 \mathrm{mg}, 0.0044 \mathrm{mmol})$ and $n \mathrm{Bu}_{4} \mathrm{NPF}_{6}(1.72 \mathrm{mg}, 0.0044 \mathrm{mmol})$ in DCE $(2 \mathrm{~mL})$ at $80{ }^{\circ} \mathrm{C}$. The reaction was stirred for 5 minutes and 4-chlorothiophenol (77 $\mathrm{mg}, 0.533 \mathrm{mmol}$ ) was added in a single portion and stirred at $80{ }^{\circ} \mathrm{C}$ for 15 minutes. Once TLC analysis indicated conversion to the product, the product was concentrated and purified by flash column chromatography $(1: 1$, EtOAc:Hex $)$ to afford a cream solid (136 mg, 88\%). RF (1:1 EtOAc/Hex) $=$ 0.50. IR $\mathrm{v}_{\max }\left(\mathrm{cm}^{-1}\right): 3162,3060,2828,1694,1469,1312$. HRMS (ESI) m/z: $[\mathrm{M}+\mathrm{H}]^{+}$Calcd for $\mathrm{C}_{20} \mathrm{H}_{15} \mathrm{CINOS} 352.0563$; found 352.0562. ${ }^{1} \mathrm{H}$ NMR (400 MHz, DMSO-d $\left.\mathrm{d}_{6}\right): \delta 9.86(\mathrm{~s}$, $1 \mathrm{H}), 7.88(\mathrm{~d}, J=7.7 \mathrm{~Hz}, 1 \mathrm{H}), 7.78(\mathrm{~d}, J=7.6 \mathrm{~Hz}, 2 \mathrm{H}), 7.63(\mathrm{dd}$, $J=10.8,4.2 \mathrm{~Hz}, 1 \mathrm{H}), 7.52-7.42(\mathrm{~m}, J=7.3 \mathrm{~Hz}, 2 \mathrm{H}), 7.42-$ $7.29(\mathrm{~m}, J=15.2,7.3 \mathrm{Z} \mathrm{Hz}, 3 \mathrm{H}), 7.21(\mathrm{~d}, J=8.5 \mathrm{~Hz}, 2 \mathrm{H}), 7.11$ $(\mathrm{d}, J=8.5 \mathrm{~Hz}, 2 \mathrm{H}) \cdot{ }^{13} \mathrm{C}\left\{{ }^{1} \mathrm{H}\right\}$ NMR $\left(101 \mathrm{MHz}, \mathrm{DMSO}-\mathrm{d}_{6}\right): \delta$ $167.8,147.6,139.2,138.4,134.9,132.4,130.3,129.0,128.9$, $128.7,128.5,126.0,124.0,122.4,75.6$.

3-((3,5-dimethylphenyl)thio)-3-phenylisoindolin-1-one (6d). To a $4 \mathrm{~mL}$ vial was added 3-hydroxy-3-phenylisoindolin-1-one (100 mg, $0.44 \mathrm{mmol}), \mathrm{Ca}\left(\mathrm{NTf}_{2}\right)_{2}(3 \mathrm{mg}, 0.0044 \mathrm{mmol})$ and $n \mathrm{Bu}_{4} \mathrm{NPF}_{6}(1.7 \mathrm{mg}, 0.0044 \mathrm{mmol})$ in DCE $(2 \mathrm{~mL})$ at $80^{\circ} \mathrm{C}$. The reaction was stirred for 5 minutes and 3,5-dimethylthiophenol (74 $\mathrm{mg}, 0.533 \mathrm{mmol}$ ) was added in a single portion and stirred at $80{ }^{\circ} \mathrm{C}$ for 15 minutes. Once TLC analysis indicated conversion to the product, the product was concentrated and purified by flash column chromatography $(1: 1$, EtOAc:Hex $)$ to afford a cream solid $(132 \mathrm{mg}, 86 \%)$. RF $(1: 1 \mathrm{EtOAc} / \mathrm{Hex})=$ 0.75. IR $v_{\max }\left(\mathrm{cm}^{-1}\right): 3173,3062,2920,2855,1699,1312$. HRMS (ESI) m/z: $[\mathrm{M}+\mathrm{H}]^{+}$Calcd for $\mathrm{C}_{22} \mathrm{H}_{20} \mathrm{NOS} 346.1266$; found 346.1263. ${ }^{1} \mathrm{H}$ NMR (400 MHz, DMSO-d $\left.{ }_{6}\right): \delta 9.74(\mathrm{~s}$, $1 \mathrm{H}), 7.86(\mathrm{~d}, J=7.7 \mathrm{~Hz}, 1 \mathrm{H}), 7.78(\mathrm{~d}, J=7.7 \mathrm{~Hz}, 2 \mathrm{H}), 7.64$ (t,
$J=7.4 \mathrm{~Hz}, 1 \mathrm{H}), 7.45(\mathrm{t}, J=7.5 \mathrm{~Hz}, 2 \mathrm{H}), 7.42-7.27(\mathrm{~m}, 2 \mathrm{H})$, $6.87(\mathrm{~s}, 1 \mathrm{H}), 6.68(\mathrm{~s}, 2 \mathrm{H}), 2.04(\mathrm{~s}, 6 \mathrm{H}),{ }^{13} \mathrm{C}\left\{{ }^{1} \mathrm{H}\right\} \mathrm{NMR}(101$ MHz, DMSO- $\left.\mathrm{d}_{6}\right): \delta 167.8,148.0,139.5,137.2,134.2,132.0$, $130.8,130.6,129.0,128.9,128.5,126.0,124.2,122.2,75.5$, 20.6 .

3-((2-methoxyphenyl)thio)-3-phenylisoindolin-1-one (6e). To a $4 \mathrm{~mL}$ vial was added 3-hydroxy-3-phenylisoindolin-1-one (100 $\mathrm{mg}, 0.44 \mathrm{mmol}), \mathrm{Ca}\left(\mathrm{NTf}_{2}\right)_{2} \quad(3 \mathrm{mg}, 0.0044 \mathrm{mmol})$ and $n \mathrm{Bu}_{4} \mathrm{NPF}_{6}(1.7 \mathrm{mg}, 0.0044 \mathrm{mmol})$ in DCE $(2 \mathrm{~mL})$ at $80^{\circ} \mathrm{C}$. The reaction was stirred for 5 minutes and 2-methoxythiophenol (93 $\mathrm{mg}, 0.666 \mathrm{mmol}$ ) was added in a single portion and stirred at 80 ${ }^{\circ} \mathrm{C}$ for 15 minutes. Once TLC analysis indicated conversion to the product, the product was concentrated and purified by flash column chromatography $(1: 1$, EtOAC:Hex $)$ to afford a cream solid $(151 \mathrm{mg}, 98 \%)$. RF $(1: 1 \mathrm{EtOAc} / \mathrm{Hex})=0.25$. IR $\mathrm{v}_{\max }\left(\mathrm{cm}^{-}\right.$ $\left.{ }^{1}\right)$ : 3190, 3065, 2925, 2830, 1695, 1471. HRMS (ESI) m/z: $[\mathrm{M}+\mathrm{H}]^{+}$Calcd for $\mathrm{C}_{21} \mathrm{H}_{18} \mathrm{NO}_{2} \mathrm{~S} 348.1058$; found $348.1060 .{ }^{1} \mathrm{H}$ NMR (400 MHz, $\left.\mathrm{CDCl}_{3}\right): \delta 7.79(\mathrm{~d}, J=7.5 \mathrm{~Hz}, 2 \mathrm{H}), 7.69(\mathrm{~d}, J$ $=7.7 \mathrm{~Hz}, 1 \mathrm{H}), 7.52(\mathrm{t}, J=7.5 \mathrm{~Hz}, 1 \mathrm{H}), 7.45(\mathrm{~d}, J=7.4 \mathrm{~Hz}, 1 \mathrm{H})$, $7.44-7.19(\mathrm{~m}, 5 \mathrm{H}), 6.91(\mathrm{~d}, J=7.3 \mathrm{~Hz}, 1 \mathrm{H}), 6.81-6.73(\mathrm{~m}$, $2 \mathrm{H}), 6.59(\mathrm{t}, J=7.4 \mathrm{~Hz}, 1 \mathrm{H}), 3.80(\mathrm{~s}, 3 \mathrm{H}) .{ }^{13} \mathrm{C}\left\{{ }^{1} \mathrm{H}\right\} \mathrm{NMR}(101$ $\left.\mathrm{MHz}, \mathrm{CDCl}_{3}\right): \delta 160.9,148.9,139.5,139.3,132.3,132.2,129.0$, $128.8,128.6,126.0,124.0,123.0,120.7,117.2,111.3,76.3$, 55.9 .

3-(2-pyrimidinethio)-3-phenylisoindolin-1-one (6f). To a $4 \mathrm{~mL}$ vial was added 3-hydroxy-3-phenylisoindolin-1-one (100 mg, $0.44 \mathrm{mmol}), \mathrm{Ca}\left(\mathrm{NTf}_{2}\right)_{2}(3 \mathrm{mg}, 0.0044 \mathrm{mmol})$ and $n \mathrm{Bu}_{4} \mathrm{NPF}_{6}$ $(1.7 \mathrm{mg}, 0.0044 \mathrm{mmol})$ in DCE $(2 \mathrm{~mL})$ at $80{ }^{\circ} \mathrm{C}$. The reaction was stirred for 5 minutes and 2-mercaptopyrimidine $(75 \mathrm{mg}$, $0.666 \mathrm{mmol}$ ) was added in a single portion and stirred at $80^{\circ} \mathrm{C}$ for 15 minutes. Once TLC analysis indicated conversion to the product, the product was concentrated and purified by flash column chromatography $(3: 1$, EtOAC:Hex) to afford a white solid $(111 \mathrm{mg}, 78 \%)$. RF $(3: 1 \mathrm{EtOAc} / \mathrm{Hex})=0.6$. IR $v_{\max }\left(\mathrm{cm}^{-}\right.$ $\left.{ }^{1}\right): 3288,3057,2924,1693,1609,1377$. HRMS (ESI) m/z: $[\mathrm{M}+\mathrm{Na}]^{+}$Calcd for $\mathrm{C}_{18} \mathrm{H}_{13} \mathrm{~N}_{3} \mathrm{OSNa}$ 342.0672; found 342.0671 . ${ }^{1} \mathrm{H}$ NMR $\left(400 \mathrm{MHz}, \mathrm{CDCl}_{3}\right): \delta .81(\mathrm{~s}, 1 \mathrm{H}), 8.42(\mathrm{~d}, J=4.9 \mathrm{~Hz}$, $2 \mathrm{H}), 7.90(\mathrm{~d}, J=7.1 \mathrm{~Hz}, 1 \mathrm{H}), 7.86-7.81(\mathrm{~m}, 2 \mathrm{H}), 7.62-7.42$ $(\mathrm{m}, 3 \mathrm{H}), 7.32-7.26(\mathrm{~m}, 2 \mathrm{H}), 7.26-7.19(\mathrm{~m}, 1 \mathrm{H}), 6.96(\mathrm{t}, J=$ $4.9 \mathrm{~Hz}, 1 \mathrm{H}) .{ }^{13} \mathrm{C}\left\{{ }^{1} \mathrm{H}\right\} \mathrm{NMR}\left(101 \mathrm{MHz}, \mathrm{CDCl}_{3}\right): \delta 171.4,169.4$, $157.5,147.7,140.2,133.1,130.2,129.7,128.8,128.3,126.2$, $124.6,123.2,117.7,74.3$

3-(2-furfurylthio)-3-phenylisoindolin-1-one (6g). To a $4 \mathrm{~mL}$ vial was added 3-hydroxy-3-phenylisoindolin-1-one $(100 \mathrm{mg}$, $0.44 \mathrm{mmol}), \mathrm{Ca}\left(\mathrm{NTf}_{2}\right)_{2}(3 \mathrm{mg}, 0.0044 \mathrm{mmol})$ and $n \mathrm{Bu}_{4} \mathrm{NPF}_{6}$ $(1.7 \mathrm{mg}, 0.0044 \mathrm{mmol})$ in DCE $(2 \mathrm{~mL})$ at $80{ }^{\circ} \mathrm{C}$. The reaction was stirred for 5 minutes and 2-furfurylthiol $(76 \mathrm{mg}, 0.666$ mmol) was added in a single portion and stirred at $80^{\circ} \mathrm{C}$ for 15 minutes. Once TLC analysis indicated conversion to the product, the product was concentrated and purified by flash column chromatography $(1: 1$, EtOAc:Hex $)$ to afford a cream solid $(143 \mathrm{mg}, 78 \%)$. RF $(1: 1 \mathrm{EtOAc} / \mathrm{Hex})=0.39$. IR $\mathrm{v}_{\max }\left(\mathrm{cm}^{-}\right.$ 1): 3170, 3061, 2926, 1692, 1467, 1148. HRMS (ESI) m/z: Calcd for $\mathrm{C}_{19} \mathrm{H}_{16} \mathrm{NO}_{2} \mathrm{~S}$ 322.0902; found 322.0901. ${ }^{1} \mathrm{H}$ NMR $\left(400 \mathrm{MHz}, \mathrm{DMSO}-\mathrm{d}_{6}\right): \delta 9.90(\mathrm{~s}, 1 \mathrm{H}), 7.78-7.66(\mathrm{~m}, J=7.2$ $\mathrm{Hz}, 4 \mathrm{H}), 7.61(\mathrm{t}, J=7.3 \mathrm{~Hz}, 1 \mathrm{H}), 7.56-7.48(\mathrm{~m}, J=7.3 \mathrm{~Hz}$, $1 \mathrm{H}), 7.46(\mathrm{~s}, 1 \mathrm{H}), 7.43-7.36(\mathrm{~m}, J=7.1 \mathrm{~Hz}, 2 \mathrm{H}), 7.36-7.26$ $(\mathrm{m}, 1 \mathrm{H}), 6.25(\mathrm{~s}, 1 \mathrm{H}), 5.92(\mathrm{~s}, 1 \mathrm{H}), 3.50(\mathrm{~d}, J=14.0 \mathrm{~Hz}, 1 \mathrm{H})$, $3.42(\mathrm{~d}, J=14.0 \mathrm{~Hz}, 1 \mathrm{H}) .{ }^{13} \mathrm{C}\left\{{ }^{1} \mathrm{H}\right\}$ NMR $(101 \mathrm{MHz}, \mathrm{DMSO}-$ $\left.\mathrm{d}_{6}\right): \delta 168.1,149.7,148.0,142.5,139.7,132.8,130.5,129.2$, $128.9,128.5,125.9,123.8,123.0,110.7,107.8,72.9,26.4$. 
3-(benzylthio)-3-phenylisoindolin-1-one (6h). To a $4 \mathrm{~mL}$ vial was added 3-hydroxy-3-phenylisoindolin-1-one (100 mg, 0.44 $\mathrm{mmol}), \mathrm{Ca}\left(\mathrm{NTf}_{2}\right)_{2}(3 \mathrm{mg}, 0.0044 \mathrm{mmol})$ and $n \mathrm{Bu}_{4} \mathrm{NPF}_{6}(1.7 \mathrm{mg}$, $0.0044 \mathrm{mmol})$ in DCE $(2 \mathrm{~mL})$ at $80{ }^{\circ} \mathrm{C}$. The reaction was stirred for 5 minutes and benzylmercaptan $(66 \mathrm{mg}, 0.53 \mathrm{mmol})$ was added in a single portion and stirred at $80{ }^{\circ} \mathrm{C}$ for 15 minutes. Once TLC analysis indicated conversion to the product, the product was concentrated and purified by flash column chromatography $(1: 1$, EtOAC:Hex $)$ to afford a cream solid (135 $\mathrm{mg}, 92 \%)$. RF $(1: 1 \mathrm{EtOAc} / \mathrm{Hex})=0.46$. IR $v_{\max }\left(\mathrm{cm}^{-1}\right): 3127$, 3056, 2843, 1694, 1609, 1491. HRMS (ESI) m/z: $[\mathrm{M}+\mathrm{H}]^{+}$ Calcd for $\mathrm{C}_{21} \mathrm{H}_{18} \mathrm{NOS} 332.1109$; found 332.1104. ${ }^{1} \mathrm{H}$ NMR (400 MHz, DMSO-d $\left.{ }_{6}\right): \delta 9.92(\mathrm{~s}, 1 \mathrm{H}), 7.84-7.66(\mathrm{~m}, 4 \mathrm{H}), 7.61(\mathrm{t}, J$ $=7.1 \mathrm{~Hz}, 1 \mathrm{H}), 7.51(\mathrm{t}, J=7.3 \mathrm{~Hz}, 1 \mathrm{H}), 7.46-7.38(\mathrm{~m}, 2 \mathrm{H})$, $7.38-7.27(\mathrm{~m}, 1 \mathrm{H}), 7.26-7.12(\mathrm{~m}, 3 \mathrm{H}), 7.11-7.03(\mathrm{~m}, 2 \mathrm{H})$, $3.45(\mathrm{~d}, J=12.2 \mathrm{~Hz}, 1 \mathrm{H}), 3.30(\mathrm{~d}, J=12.2 \mathrm{~Hz}, 1 \mathrm{H}) .{ }^{13} \mathrm{C}\left\{{ }^{1} \mathrm{H}\right\}$ NMR (101 MHz, DMSO-d $\left.{ }_{6}\right): \delta 168.1,148.2,139.9,136.5$, $132.8,130.6,129.1,128.9,128.8,128.4,127.0,125.9,123.8$, $123.0,73.1,34.1$

3-(butanethio)-3-phenylisoindolin-1-one (6i). To a $4 \mathrm{~mL}$ vial was added 3-hydroxy-3-phenylisoindolin-1-one (100 mg, 0.44 $\mathrm{mmol}), \mathrm{Ca}\left(\mathrm{NTf}_{2}\right)_{2}(3 \mathrm{mg}, 0.0044 \mathrm{mmol})$ and $n \mathrm{Bu}_{4} \mathrm{NPF}_{6}(1.7 \mathrm{mg}$, $0.0044 \mathrm{mmol})$ in DCE $(2 \mathrm{~mL})$ at $80^{\circ} \mathrm{C}$. The reaction was stirred for 5 minutes and butanethiol ( $48 \mathrm{mg}, 0.53 \mathrm{mmol}$ ) was added in a single portion and stirred at $80{ }^{\circ} \mathrm{C}$ for 15 minutes. Once TLC analysis indicated conversion to the product, the product was concentrated and purified by flash column chromatography (1:1, EtOAc:Hex) to afford a yellow oil which solidified upon standing $(120 \mathrm{mg}, 91 \%)$. RF $(1: 1 \mathrm{EtOAc} / \mathrm{Hex})=0.70$. IR $v_{\max }$ $\left(\mathrm{cm}^{-1}\right): 3185,3066,2956,1698,1310$. HRMS (ESI) $\mathrm{m} / \mathrm{z}$ : $[\mathrm{M}+\mathrm{H}]^{+}$Calcd for $\mathrm{C}_{18} \mathrm{H}_{20} \mathrm{NOS} 298.1266$; found 298.1259. ${ }^{1} \mathrm{H}$ NMR $\left(400 \mathrm{MHz}, \mathrm{CDCl}_{3}\right): \delta 7.98(\mathrm{~s}, 1 \mathrm{H}), 7.83(\mathrm{~d}, J=7.5 \mathrm{~Hz}$, $1 \mathrm{H}), 7.77-7.67(\mathrm{~m}, 2 \mathrm{H}), 7.67-7.53(\mathrm{~m}, 2 \mathrm{H}), 7.45(\mathrm{td}, J=7.4$, $0.8 \mathrm{~Hz}, 1 \mathrm{H}), 7.40-7.27(\mathrm{~m}, 3 \mathrm{H}), 2.35-2.25(\mathrm{~m}, 1 \mathrm{H}), 2.11-$ $1.98(\mathrm{~m}, 1 \mathrm{H}), 1.45-1.12(\mathrm{~m}, 4 \mathrm{H}), 0.74(\mathrm{t}, J=7.2 \mathrm{~Hz}, 3 \mathrm{H})$. ${ }^{13} \mathrm{C}\left\{{ }^{1} \mathrm{H}\right\} \mathrm{NMR}\left(101 \mathrm{MHz}, \mathrm{CDCl}_{3}\right): \delta 169.7,149.3,140.0,132.9$, $130.3,129.0,128.9,128.6,125.8,123.8,123.7,73.2,30.7,29.3$, $22.1,13.6$

3-(cyclohexanethio)-3-phenylisoindolin-1-one (6j). To a $4 \mathrm{~mL}$ vial was added 3-hydroxy-3-phenylisoindolin-1-one $(100 \mathrm{mg}$, $0.44 \mathrm{mmol}), \mathrm{Ca}\left(\mathrm{NTf}_{2}\right)_{2}(3 \mathrm{mg}, 0.0044 \mathrm{mmol})$ and $n \mathrm{Bu}_{4} \mathrm{NPF}_{6}$ $(1.7 \mathrm{mg}, 0.0044 \mathrm{mmol})$ in DCE $(2 \mathrm{~mL})$ at $80^{\circ} \mathrm{C}$. The reaction was stirred for 5 minutes and cyclohexanethiol $(77 \mathrm{mg}, 0.66$ mmol) was added in a single portion and stirred at $80^{\circ} \mathrm{C}$ for 15 minutes. Once TLC analysis indicated conversion to the product, the product was concentrated and purified by flash column chromatography $(1: 1$, EtOAc:Hex $)$ to afford a yellow oil which solidified upon standing (134 mg, 93\%). RF (1:1 EtOAc/Hex $)=0.52$. IR $v_{\max }\left(\mathrm{cm}^{-1}\right): 3156,3061,2926,1690$, 1464, 1312. HRMS (ESI) m/z: $[\mathrm{M}+\mathrm{H}]^{+}$Calcd for $\mathrm{C}_{20} \mathrm{H}_{22} \mathrm{NOS}$ 324.1422; found 324.1418. ${ }^{1} \mathrm{H}$ NMR (400 MHz, $\left.\mathrm{CDCl}_{3}\right): \delta 7.83$ $(\mathrm{d}, J=7.5 \mathrm{~Hz}, 1 \mathrm{H}), 7.75-7.68(\mathrm{~m}, 3 \mathrm{H}), 7.63(\mathrm{~d}, J=7.7 \mathrm{~Hz}$, $1 \mathrm{H}), 7.56(\mathrm{td}, J=7.6,1.0 \mathrm{~Hz}, 1 \mathrm{H}), 7.45(\mathrm{td}, J=7.5,0.9 \mathrm{~Hz}$, $1 \mathrm{H}), 7.39-7.24(\mathrm{~m}, 3 \mathrm{H}), 2.35-2.20(\mathrm{~m}, 1 \mathrm{H}), 1.91-1.75(\mathrm{~m}$, $1 \mathrm{H}), 1.68-1.55(\mathrm{~m}, 1 \mathrm{H}), 1.55-1.44(\mathrm{~m}, 1 \mathrm{H}), 1.44-1.28(\mathrm{~m}$, $3 \mathrm{H}), 1.28-0.97(\mathrm{~m}, 4 \mathrm{H}) .{ }^{13} \mathrm{C}\left\{{ }^{1} \mathrm{H}\right\} \mathrm{NMR}\left(101 \mathrm{MHz}^{\mathrm{C}} \mathrm{CDCl}_{3}\right): \delta$ $169.5,149.6,140.3,132.8,130.1,128.9,128.6,125.8,124.0$, $123.8,77.5,43.3,35.1,34.6,26.0,25.9,25.5$.
3-(4-(4,4,5,5-Tetramethyl-1,3,2-dioxaborolan-2-

yl)benzenethiol)-3-phenylisoindolin-1-one (6k). To a $4 \mathrm{~mL}$ vial was added 3-hydroxy-3-phenylisoindolin-1-one $(50 \mathrm{mg}, 0.22$ $\mathrm{mmol}), \mathrm{Ca}\left(\mathrm{NTf}_{2}\right)_{2}(3 \mathrm{mg}, 0.0022 \mathrm{mmol})$ and $n \mathrm{Bu}_{4} \mathrm{NPF}_{6}(1.7 \mathrm{mg}$, $0.0022 \mathrm{mmol})$ in DCE $(1 \mathrm{~mL})$ at $80^{\circ} \mathrm{C}$. The reaction was stirred for 5 minutes and 4-(4,4,5,5-Tetramethyl-1,3,2-dioxaborolan2-yl)benzenethiol (79 $\mathrm{mg}, 0.33 \mathrm{mmol})$ was added in a single portion and stirred at $80^{\circ} \mathrm{C}$ for 15 minutes. Once TLC analysis indicated conversion to the product, the product was concentrated and purified by flash column chromatography (1:1, EtOAc:Hex) to afford a peach solid (73 mg, 74\%). RF (1:1 EtOAc/CycHex $)=0.60$. IR v $v_{\max }\left(\mathrm{cm}^{-1}\right): 3502,3162,3067,2989$, 1697, 1358. HRMS (ESI) m/z: [M+H] $]^{+}$Calcd for $\mathrm{C}_{26} \mathrm{H}_{27} \mathrm{BNO}_{3} \mathrm{~S}$ 444.1805; found 444.1813. ${ }^{1} \mathrm{H}$ NMR (400 MHz, DMSO-d $\left.\mathrm{d}_{6}\right): \delta$ $9.88(\mathrm{~s}, 1 \mathrm{H}), 7.89(\mathrm{~d}, J=7.8 \mathrm{~Hz}, 1 \mathrm{H}), 7.78(\mathrm{~d}, J=7.4 \mathrm{~Hz}, 2 \mathrm{H})$, $7.68-7.61(\mathrm{~m}, 1 \mathrm{H}), 7.46(\mathrm{t}, J=7.5 \mathrm{~Hz}, 2 \mathrm{H}), 7.38(\mathrm{q}, J=8.1$ $\mathrm{Hz}, 4 \mathrm{H}), 7.30(\mathrm{~d}, J=7.4 \mathrm{~Hz}, 1 \mathrm{H}), 7.14(\mathrm{~d}, J=8.0 \mathrm{~Hz}, 2 \mathrm{H}), 1.25$ (s, $12 \mathrm{H}) .{ }^{13} \mathrm{C}\left\{{ }^{1} \mathrm{H}\right\}$ NMR (101 MHz, DMSO-d 6 ): $\delta 167.8,147.8$, $139.4,135.9,134.1,133.1,132.3,130.3,128.9,128.8,128.7$, $126.0,124.1,122.4,84.0,75.5,25.0,24.7,24.7$.

2-benzyl-3-(2-chloro-4-methylanilino)-2,3-dihydro-1Hisoindol-1-one (8a). To a $4 \mathrm{~mL}$ vial was added 2-benzyl-3hydroxy-2,3-dihydro-isoindol-1-one $(50 \mathrm{mg}, 0.21 \mathrm{mmol})$, $\mathrm{Ca}\left(\mathrm{NTf}_{2}\right)_{2}(13 \mathrm{mg}, 0.021 \mathrm{mmol})$ and $n \mathrm{Bu}_{4} \mathrm{NPF}_{6}(8 \mathrm{mg}, 0.021$ $\mathrm{mmol})$ in DCE $(1 \mathrm{~mL})$ at room temperature. 2-chloro-4methylaniline $(45 \mathrm{mg}, 0.31 \mathrm{mmol}$ ) was added in a single portion and the reaction was stirred at $80{ }^{\circ} \mathrm{C}$ overnight. Once TLC analysis indicated conversion to the product, the product was concentrated and purified by flash column chromatography $(0$ to $2 \%$ EtOAc:DCM) to afford the desired product as a cream solid $(48 \mathrm{mg}, 63 \%)$. RF $(1: 1 \mathrm{EtOAc} / \mathrm{Hex})=0.71$. IR $\mathrm{v}_{\max }\left(\mathrm{cm}^{-}\right.$ 1): 3361, 3031, 2924, 1683, 1615, 1520. HRMS (ESI) m/z: $[\mathrm{M}+\mathrm{H}]^{+}$Calcd for $\mathrm{C}_{22} \mathrm{H}_{20} \mathrm{ClN}_{2} \mathrm{O}$ 363.1264; found. 363.1265. ${ }^{1} \mathrm{H}$ NMR (400 MHz, DMSO-d $\left.\mathrm{d}_{6}\right): \delta 7.86-7.78(\mathrm{~m}, 1 \mathrm{H}), 7.69-7.53$ $(\mathrm{m}, 2 \mathrm{H}), 7.46(\mathrm{~d}, J=7.1 \mathrm{~Hz}, 1 \mathrm{H}), 7.33-7.22(\mathrm{~m}, 2 \mathrm{H}), 7.22-$ $7.17(\mathrm{~m}, 2 \mathrm{H}), 7.09(\mathrm{~d}, J=1.5 \mathrm{~Hz}, 1 \mathrm{H}), 6.68(\mathrm{dd}, J=8.4,1.5 \mathrm{~Hz}$, $1 \mathrm{H}), 6.25(\mathrm{~d}, J=8.2 \mathrm{~Hz}, 1 \mathrm{H}), 6.12(\mathrm{~d}, J=8.3 \mathrm{~Hz}, 1 \mathrm{H}), 6.04(\mathrm{~d}$, $J=8.2 \mathrm{~Hz}, 1 \mathrm{H}), 4.87(\mathrm{~d}, J=15.4 \mathrm{~Hz}, 1 \mathrm{H}), 4.21(\mathrm{~d}, J=15.5 \mathrm{~Hz}$, 1H), $2.10(\mathrm{~s}, 3 \mathrm{H}),{ }^{13} \mathrm{C}\left\{{ }^{1} \mathrm{H}\right\}$ NMR $\left(101 \mathrm{MHz}, \mathrm{DMSO}-\mathrm{d}_{6}\right): \delta$ $166.6,143.9,139.1,137.5,132.3,132.1,129.7,129.3,128.4$, $128.1,128.0,127.6,127.1,123.3,122.9,119.3,113.8,68.5$, $42.4,19.6$

2-benzyl-3-(2,4-difluoroanilino)-2,3-dihydro-1H-isoindol-1one $(\boldsymbol{8 b})$. To a $4 \mathrm{~mL}$ vial was added 2-benzyl-3-hydroxy-2,3dihydro-isoindol-1-one (50 mg, $0.21 \mathrm{mmol}), \mathrm{Ca}\left(\mathrm{NTf}_{2}\right)_{2}(13 \mathrm{mg}$, $0.021 \mathrm{mmol})$ and $n \mathrm{Bu}_{4} \mathrm{NPF}_{6}(8 \mathrm{mg}, 0.021 \mathrm{mmol})$ in DCE $(1 \mathrm{~mL})$ at room temperature. 2,4-difluoroaniline $(41 \mathrm{mg}, 0.31 \mathrm{mmol})$ was added in a single portion and the reaction was stirred at 80 ${ }^{\circ} \mathrm{C}$ overnight. Once TLC analysis indicated conversion to the product, the product was concentrated and purified by flash column chromatography $(1: 10$ EtOAc:Hex $)$ to afford the desired product as a colorless oil (48 mg, 68\%). RF (1:1 EtOAc/Hex $)=0.58 . I R v_{\max }\left(\mathrm{cm}^{-1}\right): 3415,2917,1694,1497$, 1113. HRMS (ESI) $\mathrm{m} / \mathrm{z}$ : $[\mathrm{M}+\mathrm{H}]^{+}$Calcd for $\mathrm{C}_{21} \mathrm{H}_{17} \mathrm{~F}_{2} \mathrm{~N}_{2} \mathrm{O}$ 351.1309; found 351.1315. . ${ }^{1} \mathrm{H}$ NMR $\left(400 \mathrm{MHz}, \mathrm{DMSO}_{6}\right): \delta$ $7.94-7.92(\mathrm{~m}, 1 \mathrm{H}), 7.57-7.53(\mathrm{~m}, 2 \mathrm{H}), 7.47-7.43(\mathrm{~m}, 1 \mathrm{H})$, $7.32-7.24(\mathrm{~m}, 3 \mathrm{H}), 7.21-7.19(\mathrm{~m}, 2 \mathrm{H}), 6.79(\mathrm{ddd}, J=11.3$, $8.4,2.8 \mathrm{~Hz}, 1 \mathrm{H}), 6.56$ (ddd, $J=4.3,3.5,1.6 \mathrm{~Hz}, 1 \mathrm{H}), 6.27(\mathrm{td}$, $J=9.3,5.4 \mathrm{~Hz}, 1 \mathrm{H}), 5.71(\mathrm{~s}, 1 \mathrm{H}), 5.26(\mathrm{~d}, J=15.1 \mathrm{~Hz}, 1 \mathrm{H})$, $4.29-4.11(\mathrm{~m}, 2 \mathrm{H}) .{ }^{13} \mathrm{C}\left\{{ }^{1} \mathrm{H}\right\}$ NMR (101 MHz, DMSO-d 6$): \delta$ $167.7,143.2$, 137.0, 132.4, 132.1, 129.8, 129.6 (dd, $J=11.4$, 
$2.9 \mathrm{~Hz}), 128.9,128.2,127.8,124.00,123.1,115.3(\mathrm{dd}, J=8.9$, $3.4 \mathrm{~Hz}), 111.2(\mathrm{~d}, J=3.7 \mathrm{~Hz}), 111.0(\mathrm{~d}, J=3.7 \mathrm{~Hz}), 104.0(\mathrm{dd}$, $J=26.4,23.5 \mathrm{~Hz}), 68.9,43.2$.

2-benzyl-3-[2-methoxy-5-(trifluoromethyl)anilino]-2,3dihydro-1H-isoindol-1-one $(\boldsymbol{8 c})$. To a $4 \mathrm{~mL}$ vial was added 2benzyl-3-hydroxy-2,3-dihydro-isoindol-1-one $(50 \mathrm{mg}, \quad 0.21$ mmol $), \mathrm{Ca}\left(\mathrm{NTf}_{2}\right)_{2}(13 \mathrm{mg}, 0.021 \mathrm{mmol})$ and $n \mathrm{Bu}_{4} \mathrm{NPF}_{6}(8 \mathrm{mg}$, $0.021 \mathrm{mmol})$ in DCE $(1 \mathrm{~mL})$ at room temperature. 2-methoxy5-(trifluoromethyl)aniline $(60 \mathrm{mg}, 0.31 \mathrm{mmol})$ was added in a single portion and the reaction was stirred at $80^{\circ} \mathrm{C}$ overnight. Once TLC analysis indicated conversion to the product, the product was concentrated and purified by flash column chromatography ( 0 to $5 \%$ EtOAc:DCM) to afford the desired product as a light brown solid (70 mg, 81\%). RF (1:20 EtOAc/DCM $)=0.33$. IR $v_{\max }\left(\mathrm{cm}^{-1}\right): 3415,2917,1694,1497$, 1113. HRMS (ESI) m/z: $[\mathrm{M}+\mathrm{H}]^{+}$Calcd for $\mathrm{C}_{23} \mathrm{H}_{19} \mathrm{~F}_{3} \mathrm{~N}_{2} \mathrm{O}_{2}$ 413.1477; found 413.1497. ${ }^{1} \mathrm{H}$ NMR (400 MHz, DMSO-d ${ }_{6}$ ): $\delta$ $7.81(\mathrm{~d}, J=6.5 \mathrm{~Hz}, 1 \mathrm{H}), 7.70-7.52(\mathrm{~m}, 2 \mathrm{H}), 7.48(\mathrm{~d}, J=6.8$ $\mathrm{Hz}, 1 \mathrm{H}), 7.28-7.05(\mathrm{~m}, 5 \mathrm{H}), 6.95-6.82(\mathrm{~m}, 2 \mathrm{H}), 6.44(\mathrm{~d}, J=$ $8.1 \mathrm{~Hz}, 1 \mathrm{H}), 6.21(\mathrm{~s}, 1 \mathrm{H}), 6.12(\mathrm{~d}, J=8.3 \mathrm{~Hz}, 1 \mathrm{H}), 4.79(\mathrm{~d}, J=$ $15.4 \mathrm{~Hz}, 1 \mathrm{H}), 4.28(\mathrm{~d}, J=15.5 \mathrm{~Hz}, 1 \mathrm{H}), 3.81(\mathrm{~s}, 3 \mathrm{H}) .{ }^{13} \mathrm{C}\left\{{ }^{1} \mathrm{H}\right\}$ NMR $\left.(101 \mathrm{MHz}, \text { DMSO-d })_{6}\right): \delta 166.6,149.6,143.7,137.4$, $135.2,132.3,132.1,129.4,128.2,127.4,126.9,123.4,122.9$, $114.6,110.0,107.5,68.2,55.8,42.7$.

2-benzyl-3-(cyclopropylamino)-2,3-dihydro-1H-isoindol-1-one $(\boldsymbol{8 d})$. To a $4 \mathrm{~mL}$ vial was added 2-benzyl-3-hydroxy-2,3dihydro-isoindol-1-one (50 mg, $0.21 \mathrm{mmol}), \mathrm{Ca}\left(\mathrm{NTf}_{2}\right)_{2}(13 \mathrm{mg}$, $0.021 \mathrm{mmol})$ and $n \mathrm{Bu}_{4} \mathrm{NPF}_{6}(8 \mathrm{mg}, 0.021 \mathrm{mmol})$ in DCE $(1 \mathrm{~mL})$ at room temperature. cyclopropylamine $(18 \mathrm{mg}, 0.31 \mathrm{mmol})$ was added in a single portion and the reaction was stirred at 80 ${ }^{\circ} \mathrm{C}$ overnight. Once TLC analysis indicated conversion to the product, the product was concentrated and purified by flash column chromatography (1:5 EtOAc:Hex, $\left.1 \% \mathrm{NEt}_{3}\right)$ to afford the desired product as a colorless oil $(39 \mathrm{mg}, 67 \%)$ RF (1:5 EtOAc/Hex $)=0.48$. IR $v_{\max }\left(\mathrm{cm}^{-1}\right): 3415,2917,1694,1497$, 1113. HRMS (ESI) m/z: $[\mathrm{M}+\mathrm{H}]^{+}$Calcd for $\mathrm{C}_{18} \mathrm{H}_{19} \mathrm{~N}_{2} \mathrm{O}$ 279.1497; found 279.1492. ${ }^{1} \mathrm{H}$ NMR (400 MHz, DMSO-d ${ }_{6}$ ): $\delta$ $7.88-7.86(\mathrm{~m}, 1 \mathrm{H}), 7.55-7.46(\mathrm{~m}, 3 \mathrm{H}), 7.33-7.24(\mathrm{~m}, 5 \mathrm{H})$, $5.30(\mathrm{~d}, J=15.0 \mathrm{~Hz}, 1 \mathrm{H}), 5.16(\mathrm{~s}, 1 \mathrm{H}), 4.27(\mathrm{~d}, J=15.0 \mathrm{~Hz}$, $1 \mathrm{H}), 2.05-2.00(\mathrm{~m}, 1 \mathrm{H}), 0.39-0.30(\mathrm{~m}, 2 \mathrm{H}), 0.22-0.08(\mathrm{~m}$, 2H). ${ }^{13} \mathrm{C}\left\{{ }^{1} \mathrm{H}\right\} \mathrm{NMR}\left(101 \mathrm{MHz}, \mathrm{DMSO}-\mathrm{d}_{6}\right): \delta 167.8,144.6$, $137.6,132.5,131.6,129.0,128.9,128.3,127.6,123.6,123.5$, $72.4,43.1,24.7,7.6,6.0$.

2-benzyl-3-(benzylamino)-2,3-dihydro-1H-isoindol-1-one (8e). To a $4 \mathrm{~mL}$ vial was added 2-benzyl-3-hydroxy-2,3-dihydroisoindol-1-one (50 mg, $0.21 \mathrm{mmol}), \mathrm{Ca}\left(\mathrm{NTf}_{2}\right)_{2}(12.5 \mathrm{mg}, 0.021$ mmol) and $n \mathrm{Bu}_{4} \mathrm{NPF}_{6}(8.1 \mathrm{mg}, 0.021 \mathrm{mmol})$ in DCE $(1 \mathrm{~mL})$ at room temperature. benzylamine $(34 \mathrm{mg}, 0.31 \mathrm{mmol}$ ) was added in a single portion and the reaction was stirred at $80{ }^{\circ} \mathrm{C}$ overnight. Once TLC analysis indicated conversion to the product, the product was concentrated and purified by flash column chromatography (1:5 EtOAc:Hex, 1\% $\left.\mathrm{NEt}_{3}\right)$ to afford the desired product as a colorless oil (64 mg, 93\%). RF (1:1 EtOAc/Hex $)=0.61$. IR $v_{\max }\left(\mathrm{cm}^{-1}\right): 3320,3029,2850,1678$, 1454, 1219. HRMS (ESI) m/z: [M+H] ${ }^{+}$Calcd for $\mathrm{C}_{22} \mathrm{H}_{21} \mathrm{~N}_{2} \mathrm{O}$ 329.1654; found 329.1652. ${ }^{1} \mathrm{H}$ NMR (400 MHz, DMSO-d ${ }_{6}$ ): $\delta$ $7.89(\mathrm{~d}, J=7.3 \mathrm{~Hz}, 1 \mathrm{H}), 7.57-7.48(\mathrm{~m}, 3 \mathrm{H}), 7.34-7.21(\mathrm{~m}$, $8 \mathrm{H}), 7.16(\mathrm{~d}, J=6.8 \mathrm{~Hz}, 2 \mathrm{H}), 5.34(\mathrm{~s}, 1 \mathrm{H}), 5.19$ (d, $J=15.0 \mathrm{~Hz}$, 1H), 4.34 (d, $J=15.0 \mathrm{~Hz}, 1 \mathrm{H}), 3.36(\mathrm{~d}, J=13.0 \mathrm{~Hz}, 1 \mathrm{H}), 3.25$ $(\mathrm{d}, J=13.0 \mathrm{~Hz}, 1 \mathrm{H}) \cdot{ }^{13} \mathrm{C}\left\{{ }^{1} \mathrm{H}\right\}$ NMR $\left(101 \mathrm{MHz}, \mathrm{DMSO}-\mathrm{d}_{6}\right): \delta$
$168.2,143.6,140.0,137.8,133.2,132.3,129.6,129.3,128.8$, $128.7,128.5,128.1,127.6,124.0,123.6,72.7,45.9,43.6$.

Benzyl (2-benzyl-3-oxo-2,3-dihydro-1H-isoindol-1yl)carbamate (12a). To a $4 \mathrm{~mL}$ vial was added 2-benzyl-3hydroxy-2,3-dihydro-isoindol-1-one $(50 \mathrm{mg}, 0.21 \mathrm{mM})$, $\mathrm{Ca}\left(\mathrm{NTf}_{2}\right)_{2}(1.3 \mathrm{mg}, 0.0021 \mathrm{mmol})$ and $n \mathrm{Bu}_{4} \mathrm{NPF}_{6}(0.8 \mathrm{mg}$, $0.0021 \mathrm{mmol})$ in DCE $(1 \mathrm{~mL})$ at $80^{\circ} \mathrm{C}$. The reaction was stirred for 1 minute and benzylcarbamate $(35 \mathrm{mg}, 0.23 \mathrm{mmol}$ ) was added in a single portion and stirred at $80{ }^{\circ} \mathrm{C}$ for 15 minutes. Once TLC analysis indicated conversion to the product, the product was concentrated and purified by flash column chromatography (1:1, EtOAc:Hex) followed by trituration with $\mathrm{Et}_{2} \mathrm{O}$ to afford the product as a white solid (70 mg, 90\%). RF $(1: 5 \mathrm{EtOAc} / \mathrm{DCM})=0.74 . \mathrm{IR} \mathrm{v}_{\max }\left(\mathrm{cm}^{-1}\right): 3306,3034,2925$, 1687, 1528, 1425. . HRMS (ESI) $\mathrm{m} / \mathrm{z}:[\mathrm{M}+\mathrm{H}]^{+}$Calcd for $\mathrm{C}_{23} \mathrm{H}_{21} \mathrm{~N}_{2} \mathrm{O}_{3} 373.1552$ found 373.1552. ${ }^{1} \mathrm{H}$ NMR $(400 \mathrm{MHz}$, DMSO-d $\left.{ }_{6}\right): \delta 8.23(\mathrm{~d}, J=9.2 \mathrm{~Hz}, 1 \mathrm{H}), 7.73(\mathrm{~d}, J=7.4 \mathrm{~Hz}, 1 \mathrm{H})$, $7.64(\mathrm{t}, J=7.3 \mathrm{~Hz}, 1 \mathrm{H}), 7.55(\mathrm{dd}, J=13.5,7.3 \mathrm{~Hz}, 2 \mathrm{H}), 7.46-$ $7.20(\mathrm{~m}, 10 \mathrm{H}), 6.09(\mathrm{~d}, J=9.1 \mathrm{~Hz}, 1 \mathrm{H}), 5.20-4.98(\mathrm{~m}, 2 \mathrm{H})$, $4.80(\mathrm{~d}, J=15.4 \mathrm{~Hz}, 1 \mathrm{H}), 4.32(\mathrm{~d}, J=15.4 \mathrm{~Hz}, 1 \mathrm{H}) .{ }^{13} \mathrm{C}\left\{{ }^{1} \mathrm{H}\right\}$ NMR $\left(101 \mathrm{MHz}, \mathrm{DMSO}-\mathrm{d}_{6}\right)$ : $\delta 166.5,156.3,143.0,137.6$, $136.7,132.3,131.7,129.4,128.4,128.0,127.8,127.7,127.2$, $123.5,122.7,65.9,65.8,43.1$

tert-butyl (2-benzyl-3-oxo-2,3-dihydro-1H-isoindol-1yl) carbamate $(\mathbf{1 2 b})$. To a $4 \mathrm{~mL}$ vial was added 2-benzyl-3hydroxy-2,3-dihydro-isoindol-1-one $(50 \mathrm{mg}, 0.21 \mathrm{mmol})$, $\mathrm{Ca}\left(\mathrm{NTf}_{2}\right)_{2}(1.3 \mathrm{mg}, 0.0021 \mathrm{mmol})$ and $n \mathrm{Bu}_{4} \mathrm{NPF}_{6}(0.8 \mathrm{mg}$, $0.0021 \mathrm{mmol})$ in DCE $(1 \mathrm{~mL})$ at $80^{\circ} \mathrm{C}$. The reaction was stirred for 1 minute and tert-butylcarbamate $(29 \mathrm{mg}, 0.25 \mathrm{mmol})$ was added in a single portion and stirred at $80{ }^{\circ} \mathrm{C}$ for 15 minutes. Once TLC analysis indicated conversion to the product, the product was concentrated and purified by flash column chromatography (1:1 EtOAC:Hex) followed by trituration with $\mathrm{Et}_{2} \mathrm{O}$ to afford the product as a white solid (61 mg, 86\%). RF $(1: 1 \mathrm{EtOAc} / \mathrm{Hex})=0.50$. IR $v_{\max }\left(\mathrm{cm}^{-1}\right): 3270,2985,2929$, 1712, 1684, 1519. HRMS (ESI) $\mathrm{m} / \mathrm{z}:[\mathrm{M}+\mathrm{H}]^{+}$Calcd for $\mathrm{C}_{20} \mathrm{H}_{23} \mathrm{~N}_{2} \mathrm{O}_{2}$ 339.1709; found 339.1696. ${ }^{1} \mathrm{H}$ NMR (400 MHz, DMSO-d $\left.)_{6}\right): \delta 7.81(\mathrm{~d}, J=9.1 \mathrm{~Hz}, 1 \mathrm{H}), 7.71(\mathrm{~d}, J=7.4 \mathrm{~Hz}, 1 \mathrm{H})$, $7.63(\mathrm{t}, J=7.2 \mathrm{~Hz}, 1 \mathrm{H}), 7.58-7.46(\mathrm{~m}, 1 \mathrm{H}), 7.39-7.18(\mathrm{~m}$, $1 \mathrm{H}), 6.03$ (d, $J=9.0 \mathrm{~Hz}, 1 \mathrm{H}), 4.83$ (d, $J=15.3 \mathrm{~Hz}, 1 \mathrm{H}), 4.28$ $(\mathrm{d}, J=15.5 \mathrm{~Hz}, 1 \mathrm{H}), 1.39(\mathrm{~s}, 1 \mathrm{H}) .{ }^{13} \mathrm{C}\left\{{ }^{1} \mathrm{H}\right\}$ NMR $(101 \mathrm{MHz}$, DMSO-d $\left.{ }_{6}\right): \delta 166.6,155.6,143.3,137.8,132.2,131.7,129.3$, $128.5,127.6,127.1,123.5,122.6,79.0,65.5,43.1,28.1$.

$N$-(2-benzyl-3-oxo-2,3-dihydro-1H-isoindol-1-yl)benzamide (12c). To a $4 \mathrm{~mL}$ vial was added 2-benzyl-3-hydroxy-2,3dihydro-isoindol-1-one $(50 \mathrm{mg}, 0.21 \mathrm{mmol}) \mathrm{Ca}\left(\mathrm{NTf}_{2}\right)_{2}(6.3 \mathrm{mg}$, $0.0104 \mathrm{mmol})$ and $n \mathrm{Bu}_{4} \mathrm{NPF}_{6}(4 \mathrm{mg}, 0.0104 \mathrm{mmol})$ in DCE (1 $\mathrm{mL})$ at $80{ }^{\circ} \mathrm{C}$. The reaction was stirred for 1 minute and benzamide ( $38 \mathrm{mg}, 0.3 \mathrm{mmol}$ ) was added in a single portion and stirred at $80{ }^{\circ} \mathrm{C}$ for 15 minutes. Once TLC analysis indicated conversion to the product, the product was concentrated and purified by flash column chromatography $(1: 1$, EtOAc:Hex) to afford a white solid (68 mg, 95\%). RF (1:1 EtOAc/Hex $)=0.41$. IR $v_{\max }\left(\mathrm{cm}^{-1}\right): 3548,3278,3031,2933,1705,1270$. HRMS (ESI) $\mathrm{m} / \mathrm{z}$ : $[\mathrm{M}+\mathrm{H}]^{+}$Calcd for $\mathrm{C}_{22} \mathrm{H}_{19} \mathrm{~N}_{2} \mathrm{O}_{2}$ 343.1447; found 343.1441. ${ }^{1} \mathrm{H}$ NMR (400 MHz, DMSO-d $\left.{ }_{6}\right): \delta 9.20(\mathrm{~d}, J=8.9$ $\mathrm{Hz}, 1 \mathrm{H}), 7.82$ (d, $J=7.6 \mathrm{~Hz}, 2 \mathrm{H}), 7.78$ (d, $J=7.3 \mathrm{~Hz}, 1 \mathrm{H}), 7.71$ $-7.51(\mathrm{~m}, 4 \mathrm{H}), 7.45(\mathrm{t}, J=7.6 \mathrm{~Hz}, 2 \mathrm{H}), 7.38-7.14(\mathrm{~m}, 5 \mathrm{H})$, $6.61(\mathrm{~d}, J=8.9 \mathrm{~Hz}, 1 \mathrm{H}), 4.85(\mathrm{~d}, J=15.4 \mathrm{~Hz}, 1 \mathrm{H}), 4.37(\mathrm{~d}, J=$ $15.4 \mathrm{~Hz}, 1 \mathrm{H}) .{ }^{13} \mathrm{C}\left\{{ }^{1} \mathrm{H}\right\}$ NMR $\left(101 \mathrm{MHz}, \mathrm{DMSO}-\mathrm{d}_{6}\right): \delta 167.3$, 
$166.9,143,137.7,133.3,132.3,131.9,131.9,129.4,128.5$, $128.3,127.7,127.6,127.2,123.6,122.8,63.8,43.2$.

2-benzyl-3-(2-oxo-1,3-oxazolidin-3-yl)-2,3-dihydro-1Hisoindol-1-one $(\mathbf{1 2 d})$. To a $4 \mathrm{~mL}$ vial was added 2-benzyl-3hydroxy-2,3-dihydro-isoindol-1-one $(50 \mathrm{mg}, 0.21 \mathrm{mmol})$, $\mathrm{Ca}\left(\mathrm{NTf}_{2}\right)_{2}(1.3 \mathrm{mg}, 0.0021 \mathrm{mmol})$ and $n \mathrm{Bu}_{4} \mathrm{NPF}_{6}(1 \mathrm{mg}, 0.0021$ $\mathrm{mmol})$ in DCE $(1 \mathrm{~mL})$ at $80{ }^{\circ} \mathrm{C}$. The reaction was stirred for 1 minute and 2-oxazolidinone $(27 \mathrm{mg}, 0.31 \mathrm{mmol})$ was added in a single portion and stirred at $80{ }^{\circ} \mathrm{C}$ for 15 minutes. Once TLC analysis indicated conversion to the product, the product was concentrated and purified by flash column chromatography (1:9, EtOAc:DCM) to afford a white semi-solid (49 mg, 76\%). $\mathrm{RF}(1: 5 \mathrm{EtOAc} / \mathrm{DCM})=0.64 . \mathrm{IR} v_{\max }\left(\mathrm{cm}^{-1}\right): 2972,1755,1701$, 1404, 1215. HRMS (ESI) m/z: $[\mathrm{M}+\mathrm{H}]^{+}$Calcd for $\mathrm{C}_{18} \mathrm{H}_{17} \mathrm{~N}_{2} \mathrm{O}_{3}$ 309.1239; found 309.1246. ${ }^{1} \mathrm{H} \mathrm{NMR}\left(400 \mathrm{MHz}, \mathrm{CDCl}_{3}\right): \delta 7.91$ $(\mathrm{d}, J=6.9 \mathrm{~Hz}, 1 \mathrm{H}), 7.64-7.53(\mathrm{~m}, 2 \mathrm{H}), 7.46(\mathrm{~d}, J=6.9 \mathrm{~Hz}$, $2 \mathrm{H}), 7.41(\mathrm{~d}, J=7.2 \mathrm{~Hz}, 1 \mathrm{H}), 7.38-7.27(\mathrm{~m}, 3 \mathrm{H}), 6.42(\mathrm{~s}, 1 \mathrm{H})$, $4.96(\mathrm{~d}, J=14.7 \mathrm{~Hz}, 1 \mathrm{H}), 4.47(\mathrm{~d}, J=14.7 \mathrm{~Hz}, 1 \mathrm{H}), 4.06(\mathrm{td}, J$ $=9.0,6.5 \mathrm{~Hz}, 1 \mathrm{H}), 3.53(\mathrm{td}, J=9.0,7.2 \mathrm{~Hz}, 1 \mathrm{H}), 2.75(\mathrm{dd}, J=$ $15.9,8.8 \mathrm{~Hz}, 1 \mathrm{H}), 2.54$ (td, $J=9.0,6.5 \mathrm{~Hz}, 1 \mathrm{H}) .{ }^{13} \mathrm{C}\left\{{ }^{1} \mathrm{H}\right\} \mathrm{NMR}$ $\left(101 \mathrm{MHz} \mathrm{CDCl}_{3}\right): \delta 167.5,158.2,139.7,137.4,132.7,132.4$, $130.3,128.9,128.8,128.0,124.3,123.0,68.7,62.2,45.1,38.8$.

N-(2-benzyl-3-oxo-2,3-dihydro-1H-isoindol-1-yl)-4methylbenzene-1-sulfonamide (14a). To a $4 \mathrm{~mL}$ vial was added 2-benzyl-3-hydroxy-2,3-dihydro-isoindol-1-one (50 mg, 0.21 $\mathrm{mmol}), \mathrm{Ca}\left(\mathrm{NTf}_{2}\right)_{2}(6.3 \mathrm{mg}, 0.0104 \mathrm{mmol})$ and $n \mathrm{Bu}_{4} \mathrm{NPF}_{6}(4 \mathrm{mg}$, $0.0104 \mathrm{mmol})$ in DCE $(1 \mathrm{~mL})$ at $80^{\circ} \mathrm{C}$. The reaction was stirred for 1 minute and $p$-toluenesulfonamide $(36 \mathrm{mg}, 0.21 \mathrm{mmol})$ was added in a single portion and stirred at $80{ }^{\circ} \mathrm{C}$ overnight. Once TLC analysis indicated conversion to the product, hexane ( 2 $\mathrm{mL})$ was added and the product was collected by filtration as a white solid (68 mg, 85\%). RF (1:5 EtOAc/DCM) $=0.57$. IR $v_{\max }$ $\left(\mathrm{cm}^{-1}\right): 3125,2935,2884,1674,1495,1162$. HRMS (ESI) m/z: $[\mathrm{M}+\mathrm{H}]^{+}$Calcd for $\mathrm{C}_{22} \mathrm{H}_{21} \mathrm{~N}_{2} \mathrm{O}_{3} \mathrm{~S} 393.1273$; found $393.1276 .{ }^{1} \mathrm{H}$ NMR (400 MHz, DMSO-d $\left.{ }_{6}\right): \delta 8.94(\mathrm{~d}, J=8.8 \mathrm{~Hz}, 1 \mathrm{H}), 7.72$ $(\mathrm{dd}, J=25.2,6.9 \mathrm{~Hz}, 3 \mathrm{H}), 7.58-7.46(\mathrm{~m}, 2 \mathrm{H}), 7.43$ (d, $J=7.9$ $\mathrm{Hz}, 2 \mathrm{H}), 7.35-7.20(\mathrm{~m}, 3 \mathrm{H}), 7.16(\mathrm{~d}, J=7.2 \mathrm{~Hz}, 2 \mathrm{H}), 6.73(\mathrm{~d}$, $J=6.2 \mathrm{~Hz}, 1 \mathrm{H}), 5.78(\mathrm{~d}, J=8.7 \mathrm{~Hz}, 1 \mathrm{H}), 4.82(\mathrm{~d}, J=15.6 \mathrm{~Hz}$, $1 \mathrm{H}), 4.22(\mathrm{~d}, J=15.5 \mathrm{~Hz}, 1 \mathrm{H}), 2.44(\mathrm{~s}, 3 \mathrm{H}) .{ }^{13} \mathrm{C}\left\{{ }^{1} \mathrm{H}\right\} \mathrm{NMR}(101$ MHz, DMSO-d $\left.\mathrm{d}_{6}\right): \delta 166.3,143.3,142.7,139.0,137.3,132.3$, $131.2,123.0,129.7,128.4,127.50,127.1,126.4,123.1,122.8$, $67.6,42.3,21.1$.

N-(2-benzyl-3-oxo-2,3-dihydro-1H-isoindol-1-yl)-4-

methoxybenzene-1-sulfonamide (14b). To a $4 \mathrm{~mL}$ vial was added 2-benzyl-3-hydroxy-2,3-dihydro-isoindol-1-one (50 mg, $0.21 \mathrm{mmol}), \mathrm{Ca}\left(\mathrm{NTf}_{2}\right)_{2}(6.27 \mathrm{mg}, 0.0104 \mathrm{mmol})$ and $n \mathrm{Bu}_{4} \mathrm{NPF}_{6}$ $(4.05 \mathrm{mg}, 0.0104 \mathrm{mmol})$ in DCE $(1 \mathrm{~mL})$ at $80^{\circ} \mathrm{C}$. The reaction was stirred for 1 minute and 4-methoxybenezenesulfonamide $(39 \mathrm{mg}, 0.21 \mathrm{mmol})$ was added in a single portion and stirred at $80^{\circ} \mathrm{C}$ overnight. Once TLC analysis indicated conversion to the product, hexane $(2 \mathrm{~mL})$ was added and the product was collected by filtration as a white solid (78 mg, 91\%). RF (1:5 EtOAc/DCM $)=0.30$. IR $v_{\max }\left(\mathrm{cm}^{-1}\right): 3130,2929,2843,1677$, 1594, 1495. HRMS (ESI) m/z: $[\mathrm{M}+\mathrm{H}]^{+}$Calcd for $\mathrm{C}_{22} \mathrm{H}_{21} \mathrm{~N}_{2} \mathrm{O}_{4} \mathrm{~S}$
409.1222; found 409.1227. ${ }^{1} \mathrm{H}$ NMR (400 MHz, DMSO-d $\left.\mathrm{d}_{6}\right): \delta$ $8.97-8.76(\mathrm{~m}, 1 \mathrm{H}), 7.80(\mathrm{~d}, J=8.5 \mathrm{~Hz}, 2 \mathrm{H}), 7.70(\mathrm{~s}, 1 \mathrm{H}), 7.57$ $-7.47(\mathrm{~m}, 2 \mathrm{H}), 7.35-7.09(\mathrm{~m}, 7 \mathrm{H}), 6.77(\mathrm{~s}, 1 \mathrm{H}), 5.86-5.65$ (m, 1H), 4.82 (d, $J=15.6 \mathrm{~Hz}, 1 \mathrm{H}), 4.24(\mathrm{~d}, J=15.5 \mathrm{~Hz}, 1 \mathrm{H})$, $3.87(\mathrm{~s}, 3 \mathrm{H}) .{ }^{13} \mathrm{C}\left\{{ }^{1} \mathrm{H}\right\}$ NMR $\left(101 \mathrm{MHz}, \mathrm{DMSO}-\mathrm{d}_{6}\right): \delta 166.3$, $162.5,142.8,137.4,133.5,132.3,131.2,129.7,128.6,128.4$, $127.5,127.1,123.2,122.8,114.6,67.5,55.8,42.3$.

$\mathrm{N}$-(2-benzyl-3-oxo-2,3-dihydro-1H-isoindol-1-yl)-2-

(trifluoromethoxy)benzene-1-sulfonamide (14c). To a $4 \mathrm{~mL}$ vial was added 2-benzyl-3-hydroxy-2,3-dihydro-isoindol-1-one (50 $\mathrm{mg}, 0.21 \mathrm{mmol}), \mathrm{Ca}\left(\mathrm{NTf}_{2}\right)_{2}(6.2 \mathrm{mg}, 0.0104 \mathrm{mmol})$ and $n \mathrm{Bu}_{4} \mathrm{NPF}_{6}(4 \mathrm{mg}, 0.0104 \mathrm{mmol})$ in DCE $(1 \mathrm{~mL})$ at $80{ }^{\circ} \mathrm{C}$. The reaction was stirred for 1 minute and 2(trifluoromethoxy)benezenesulfonamide (55 $\mathrm{mg}, 0.23 \mathrm{mmol}$ ) was added in a single portion and stirred at $80{ }^{\circ} \mathrm{C}$ overnight. Once TLC analysis indicated conversion to the product, hexane $(2 \mathrm{~mL})$ was added and the product was collected by filtration as a white solid $(75 \mathrm{mg}, 78 \%)$. RF $(1: 5 \mathrm{EtOAc} / \mathrm{DCM})=0.56 . \mathrm{IR}$ $v_{\max }\left(\mathrm{cm}^{-1}\right): 3106,3063,1687,1592,1444,1163$. HRMS (ESI) $\mathrm{m} / \mathrm{z}:[\mathrm{M}+\mathrm{H}]^{+}$Calcd for $\mathrm{C}_{22} \mathrm{H}_{18} \mathrm{~F}_{3} \mathrm{~N}_{2} \mathrm{O}_{4} \mathrm{~S}$ 463.0939; found 463.0930. ${ }^{1} \mathrm{H}$ NMR (400 MHz, DMSO- $\left.\mathrm{d}_{6}\right): \delta 9.28(\mathrm{~d}, J=8.8$ $\mathrm{Hz}, 1 \mathrm{H}), 8.03-7.81(\mathrm{~m}, 2 \mathrm{H}), 7.80-7.61(\mathrm{~m}, 2 \mathrm{H}), 7.61-7.46$ $(\mathrm{m}, 3 \mathrm{H}), 7.42-7.19(\mathrm{~m}, 3 \mathrm{H}), 7.12(\mathrm{~d}, J=6.8 \mathrm{~Hz}, 2 \mathrm{H}), 7.06-$ $6.88(\mathrm{~m}, 1 \mathrm{H}), 5.79(\mathrm{~d}, J=8.7 \mathrm{~Hz}, 1 \mathrm{H}), 4.90(\mathrm{~d}, J=15.6 \mathrm{~Hz}$, $1 \mathrm{H}), 4.17(\mathrm{~d}, J=15.6 \mathrm{~Hz}, 1 \mathrm{H}) .{ }^{13} \mathrm{C}\left\{{ }^{1} \mathrm{H}\right\} \mathrm{NMR}(101 \mathrm{MHz}$, DMSO-d $\left.{ }_{6}\right): \delta 166.4,144.9,142.5,137.1,135.5,133.6,132.4$, $131.3,130.1,129.8,128.5,127.8,127.3,127.2,123.0,122.9$, 121.1, 67.5, 42.2.

$N$-(2-benzyl-3-oxo-2,3-dihydro-1H-isoindol-1-yl)-3-

chlorobenzene-1-sulfonamide (14d). To a $4 \mathrm{~mL}$ vial was added 2-benzyl-3-hydroxy-2,3-dihydro-isoindol-1-one $(50 \mathrm{mg}, 0.21$ $\mathrm{mmol}), \mathrm{Ca}\left(\mathrm{NTf}_{2}\right)_{2}(6.3 \mathrm{mg}, 0.0104 \mathrm{mmol})$ and $n \mathrm{Bu}_{4} \mathrm{NPF}_{6}(4 \mathrm{mg}$, $0.0104 \mathrm{mmol})$ in DCE $(1 \mathrm{~mL})$ at $80^{\circ} \mathrm{C}$. The reaction was stirred for 1 minute and 3-chloro-benezenesulfonamide (44 mg, 0.23 mmol) was added in a single portion and stirred at $80^{\circ} \mathrm{C}$ for $2 \mathrm{~h}$. Once TLC analysis indicated conversion to the product, hexane $(2 \mathrm{~mL})$ was added and the product was collected by filtration as a white solid $(65 \mathrm{mg}, 75 \%)$. RF $(1: 5 \mathrm{EtOAc} / \mathrm{DCM})=0.60$. IR $v_{\max }\left(\mathrm{cm}^{-1}\right): 3181,3056,1687,1494,1468,1157$. HRMS (ESI) $\mathrm{m} / \mathrm{z}:[\mathrm{M}+\mathrm{H}]^{+}$Calcd for $\mathrm{C}_{21} \mathrm{H}_{18} \mathrm{ClN}_{2} \mathrm{O}_{3} \mathrm{~S}$ 413.0727; found 413.0727. ${ }^{1} \mathrm{H}$ NMR (400 MHz, DMSO-d $\left.{ }_{6}\right): \delta 9.19(\mathrm{~d}, J=8.7$ $\mathrm{Hz}, 1 \mathrm{H}), 7.89-7.78(\mathrm{~m}, 3 \mathrm{H}), 7.76-7.64(\mathrm{~m}, 2 \mathrm{H}), 7.53$ (p, $J=$ $8.1 \mathrm{~Hz}, 2 \mathrm{H}), 7.36-7.22(\mathrm{~m}, 3 \mathrm{H}), 7.18(\mathrm{~d}, J=7.3 \mathrm{~Hz}, 2 \mathrm{H}), 6.76$ $(\mathrm{d}, J=6.2 \mathrm{~Hz}, 1 \mathrm{H}), 5.90(\mathrm{~d}, J=8.7 \mathrm{~Hz}, 1 \mathrm{H}), 4.83(\mathrm{~d}, J=15.6$ $\mathrm{Hz}, 1 \mathrm{H}), 4.24(\mathrm{~d}, J=15.6 \mathrm{~Hz}, 1 \mathrm{H}) .{ }^{13} \mathrm{C}\left\{{ }^{1} \mathrm{H}\right\} \mathrm{NMR}(101 \mathrm{MHz}$, DMSO-d $\left.{ }_{6}\right): \delta 166.3,143.7,142.4,137.2,134.1,133.0,132.3$, $131.9,131.2,129.8,128.5,127.5,127.2,126.1,125.0,123.2$, $122.9,67.7,42.7$.

N-(2-benzyl-3-oxo-2,3-dihydro-1H-isoindol-1-yl)-3-

fluorobenzene-1-sulfonamide (14e). To a $4 \mathrm{~mL}$ vial was added 2-benzyl-3-hydroxy-2,3-dihydro-isoindol-1-one (50 mg, 0.21 $\mathrm{mmol}), \mathrm{Ca}\left(\mathrm{NTf}_{2}\right)_{2}(6.3 \mathrm{mg}, 0.0104 \mathrm{mmol})$ and $n \mathrm{Bu}_{4} \mathrm{NPF}_{6}(4 \mathrm{mg}$, $0.0104 \mathrm{mmol})$ in DCE $(1 \mathrm{~mL})$ at $80^{\circ} \mathrm{C}$. The reaction was stirred for 1 minute and 3-fluorobenezenesulfonamide (40 mg, 0.23 mmol) was added in a single portion and stirred at $80{ }^{\circ} \mathrm{C}$ overnight. Once TLC analysis indicated conversion to the product, hexane $(2 \mathrm{~mL})$ was added and the product was collected by filtration as a white solid (65 mg, 68\%). RF (1:5 EtOAc/DCM $)=0.60$. IR $v_{\max }\left(\mathrm{cm}^{-1}\right): 3197,3072,2936,1685$, 1494, 1345. HRMS (ESI) $\mathrm{m} / \mathrm{z}: \quad[\mathrm{M}+\mathrm{H}]^{+}$Calcd for $\mathrm{C}_{21} \mathrm{H}_{18} \mathrm{FN}_{2} \mathrm{O}_{3} \mathrm{~S}$ 397.1022; found 397.1023. ${ }^{1} \mathrm{H}$ NMR (400 MHz, 
DMSO-d $\left.{ }_{6}\right): \delta 9.17(\mathrm{~d}, J=8.8 \mathrm{~Hz}, 1 \mathrm{H}), 7.80-7.57(\mathrm{~m}, 5 \mathrm{H}), 7.52$ $(\mathrm{p}, J=7.7 \mathrm{~Hz}, 2 \mathrm{H}), 7.35-7.14(\mathrm{~m}, 5 \mathrm{H}), 6.73(\mathrm{~d}, J=6.0 \mathrm{~Hz}$, $1 \mathrm{H}), 5.90(\mathrm{~d}, J=8.8 \mathrm{~Hz}, 1 \mathrm{H}), 4.83(\mathrm{~d}, J=15.6 \mathrm{~Hz}, 1 \mathrm{H}), 4.25$ $(\mathrm{d}, J=15.6 \mathrm{~Hz}, 1 \mathrm{H}) .{ }^{13} \mathrm{C}\left\{{ }^{1} \mathrm{H}\right\}$ NMR $\left(101 \mathrm{MHz}, \mathrm{DMSO}-\mathrm{d}_{6}\right): \delta$ $166.3,163.1,160.6,143.9$ (d, $J=6.6 \mathrm{~Hz}), 142.5,137.3$, 132.4, $132.1(\mathrm{~d}, J=7.9 \mathrm{~Hz}), 131.2,129.8,128.5,127.5,127.1,123.1$, $122.9,122.6,120.2(\mathrm{~d}, J=21.1 \mathrm{~Hz}), 113.6(\mathrm{~d}, J=24.5 \mathrm{~Hz})$, $67.7,42.5$

N-(2-benzyl-3-oxo-2,3-dihydro-1H-isoindol-1-yl)-2,4difluorobenzene-1-sulfonamide (14f). To a $4 \mathrm{~mL}$ vial was added 2-benzyl-3-hydroxy-2,3-dihydro-isoindol-1-one (43 mg, 0.18 $\mathrm{mmol}), \mathrm{Ca}\left(\mathrm{NTf}_{2}\right)_{2}(6 \mathrm{mg}, 0.00906 \mathrm{mmol})$ and $n \mathrm{Bu}_{4} \mathrm{NPF}_{6}(4 \mathrm{mg}$, $0.0906 \mathrm{mmol})$ in DCE $(1 \mathrm{~mL})$ at $80{ }^{\circ} \mathrm{C}$. The reaction was stirred for 1 minute and 2,4-difluorobenezenesulfonamide $(35 \mathrm{mg}$, $0.18 \mathrm{mmol}$ ) was added in a single portion and stirred at $80{ }^{\circ} \mathrm{C}$ overnight. Once TLC analysis indicated conversion to the product, hexane $(2 \mathrm{~mL})$ was added and the product was collected by filtration as a white solid (45 mg, 60\%). RF (1:5 EtOAc/DCM $)=0.69$. IR $v_{\max }\left(\mathrm{cm}^{-1}\right): 3541,3265,3091,1681$, 1601, 1418. HRMS (ESI) m/z: $[\mathrm{M}+\mathrm{H}]^{+}$Calcd for $\mathrm{C}_{21} \mathrm{H}_{17} \mathrm{~F}-$ ${ }_{2} \mathrm{~N}_{2} \mathrm{O}_{3} \mathrm{~S}$ 415.0928; found 415.0924. ${ }^{1} \mathrm{H}$ NMR $(400 \mathrm{MHz}$,

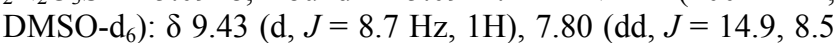
$\mathrm{Hz}, 1 \mathrm{H}), 7.76-7.67(\mathrm{~m}, 1 \mathrm{H}), 7.66-7.50$ (m, 3H), $7.35-7.20$ $(\mathrm{m}, 5 \mathrm{H}), 7.15(\mathrm{~d}, J=6.9 \mathrm{~Hz}, 2 \mathrm{H}), 7.06(\mathrm{~d}, J=6.9 \mathrm{~Hz}, 1 \mathrm{H}), 5.79$ $(\mathrm{d}, J=8.6 \mathrm{~Hz}, 1 \mathrm{H}), 4.89(\mathrm{~d}, J=15.8 \mathrm{~Hz}, 1 \mathrm{H}), 4.23(\mathrm{~d}, J=15.8$ $\mathrm{Hz}, 1 \mathrm{H}) .{ }^{13} \mathrm{C}\left\{{ }^{1} \mathrm{H}\right\}$ NMR $\left(101 \mathrm{MHz}, \mathrm{DMSO}-\mathrm{d}_{6}\right): \delta 166.5,166.5$, $164.0(\mathrm{~d}, J=11.6 \mathrm{~Hz}), 160.1(\mathrm{~d}, J=13.6 \mathrm{~Hz}), 157.5(\mathrm{~d}, J=13.5$ $\mathrm{Hz}), 142.4,137.1,132.5,131.4$ (d, $J=10.8 \mathrm{~Hz}), 131.3,129.8$, $128.5,127.2,127.2,126.2(\mathrm{dd}, J=13.9,3.4 \mathrm{~Hz}), 123.0(\mathrm{~d}, J=$ $24.2 \mathrm{~Hz}), 112.4(\mathrm{~d}, J=22.7 \mathrm{~Hz}), 67.5,42.3$.

$\mathrm{N}$-(2-benzyl-3-oxo-2,3-dihydro-1H-isoindol-1-yl)thiophene-2sulfonamide $(\mathbf{1 4 g})$. To a $4 \mathrm{~mL}$ vial was added 2-benzyl-3hydroxy-2,3-dihydro-isoindol-1-one $(50 \mathrm{mg}, 0.21 \mathrm{mmol})$, $\mathrm{Ca}\left(\mathrm{NTf}_{2}\right)_{2}(6.3 \mathrm{mg}, 0.0104 \mathrm{mmol})$ and $n \mathrm{Bu}_{4} \mathrm{NPF}_{6}(4 \mathrm{mg}, 0.0104$ $\mathrm{mmol})$ in DCE $(1 \mathrm{~mL})$ at $80{ }^{\circ} \mathrm{C}$. The reaction was stirred for 1 minute and 2-thiophenesulfonamide ( $38 \mathrm{mg}, 0.23 \mathrm{mmol}$ ) was added in a single portion and stirred at $80{ }^{\circ} \mathrm{C}$ overnight. Once TLC analysis indicated conversion to the product, hexane ( 2 $\mathrm{mL})$ was added and the product was collected by filtration as a white solid (62 mg, 77\%). RF (1:5 EtOAc/DCM) $=0.58$. IR $v_{\max }$ $\left(\mathrm{cm}^{-1}\right): 3345,3105,2894,1664,1467,1198 \ldots$ HRMS (ESI) m/z: $[\mathrm{M}+\mathrm{H}]^{+}$Calcd for $\mathrm{C}_{19} \mathrm{H}_{17} \mathrm{~N}_{2} \mathrm{O}_{3} \mathrm{~S}_{2} 385.0681$; found $385.0680 .{ }^{1} \mathrm{H}$ NMR (400 MHz, DMSO-d $\left.)_{6}\right): \delta 9.25(\mathrm{~d}, J=8.8 \mathrm{~Hz}, 1 \mathrm{H}), 8.04$ $(\mathrm{d}, J=4.8 \mathrm{~Hz}, 1 \mathrm{H}), 7.75-7.60(\mathrm{~m}, 2 \mathrm{H}), 7.56-7.45(\mathrm{~m}, 2 \mathrm{H})$, $7.37-7.17(\mathrm{~m}, 6 \mathrm{H}), 6.83-6.61(\mathrm{~m}, 1 \mathrm{H}), 5.84(\mathrm{~d}, J=8.8 \mathrm{~Hz}$, $1 \mathrm{H}), 4.85(\mathrm{~d}, J=15.5 \mathrm{~Hz}, 1 \mathrm{H}), 4.29(\mathrm{~d}, J=15.6 \mathrm{~Hz}, 1 \mathrm{H})$. ${ }^{13} \mathrm{C}\left\{{ }^{1} \mathrm{H}\right\}$ NMR (101 MHz, DMSO-d $\left.{ }_{6}\right): \delta 166.3,142.7,142.5$, 137.4, 133.4, 132.4, 132.4, 131.2, 129.8, 128.5, 128.1, 127.6, $127.1,123.1,122.9,67.7,42.5$.

N-(2-benzyl-3-oxo-2,3-dihydro-1H-isoindol-1yl)cyclopropanesulfonamide (14h). To a $4 \mathrm{~mL}$ vial was added 2-benzyl-3-hydroxy-2,3-dihydro-isoindol-1-one (50 mg, 0.21 $\mathrm{mmol}), \mathrm{Ca}\left(\mathrm{NTf}_{2}\right)_{2}(6.3 \mathrm{mg}, 0.0104 \mathrm{mmol})$ and $n \mathrm{Bu}_{4} \mathrm{NPF}_{6}(4 \mathrm{mg}$, $0.0104 \mathrm{mmol})$ in DCE $(1 \mathrm{~mL})$ at $80^{\circ} \mathrm{C}$. The reaction was stirred for 1 minute and cyclopropanesulfonamide $(28 \mathrm{mg}, 0.23 \mathrm{mmol})$ was added in a single portion and stirred at $80{ }^{\circ} \mathrm{C}$ overnight. Once TLC analysis indicated conversion to the product, hexane $(2 \mathrm{~mL}$ ) was added and the product was collected by filtration as a white solid (44 mg, 61\%). RF (1:5 EtOAc/DCM) = 0.35. IR $\mathrm{v}_{\max }\left(\mathrm{cm}^{-1}\right): 3387,3277,3125,1671,1470,1295$. HRMS (ESI) $\mathrm{m} / \mathrm{z}: \quad[\mathrm{M}+\mathrm{H}]^{+}$Calcd for $\mathrm{C}_{18} \mathrm{H}_{19} \mathrm{~N}_{2} \mathrm{O}_{3} \mathrm{~S}$ 343.1116; found
343.1118.. ${ }^{1} \mathrm{H}$ NMR (400 MHz, DMSO-d $\left.{ }_{6}\right): \delta 8.48(\mathrm{~s}, 1 \mathrm{H}), 7.80$ $-7.65(\mathrm{~m}, 3 \mathrm{H}), 7.65-7.54(\mathrm{~m}, 1 \mathrm{H}), 7.42-7.28(\mathrm{~m}, 4 \mathrm{H}), 7.28$ $-7.17(\mathrm{~m}, 1 \mathrm{H}), 5.88(\mathrm{~s}, 1 \mathrm{H}), 4.92(\mathrm{~d}, J=15.5 \mathrm{~Hz}, 1 \mathrm{H}), 4.42(\mathrm{~d}$, $J=15.6 \mathrm{~Hz}, 1 \mathrm{H}), 3.08-2.80(\mathrm{~m}, 1 \mathrm{H}), 1.13-0.97(\mathrm{~m}, 2 \mathrm{H}), 0.96$ $-0.83(\mathrm{~m}, 2 \mathrm{H}) .{ }^{13} \mathrm{C}\left\{{ }^{1} \mathrm{H}\right\}$ NMR $\left(101 \mathrm{MHz}, \mathrm{DMSO}-\mathrm{d}_{6}\right): \delta 166.3$, $143.2,137.7,132.4,131.3,129.7,128.5,127.7,127.1,124.0$, $122.7,67.7,42.2,31.6,5.6,5.3$.

\section{Supporting Information}

The Supporting Information is available free of charge on the ACS Publications website.

PDF copies of ${ }^{1} \mathrm{H}$ and ${ }^{13} \mathrm{C}$

\section{AUTHOR INFORMATION}

\section{Corresponding Author}

* E-mail:m.mclaughlin@mmu.ac.uk

\section{ACKNOWLEDGMENT}

We thank Manchester Metropolitan University for startup funding and infrastructural support

\section{REFERENCES}

1. Wu, P.; Nielsen, T. E., Scaffold Diversity from $\mathrm{N}$ Acyliminium Ions. Chem. Rev. 2017, 117, 7811-7856.

2. Maryanoff, B. E.; Zhang, H. C.; Cohen, J. H.; Turchi, I. J.; Maryanoff, C. A., Cyclizations of $\mathrm{N}$-acyliminium ions. Chem. Rev. 2004, 104, 1431.

3. Germer, W.; Stalling, T.; Kramer, U.; Saak, W.; Martens, J., A Novel Rearrangement Reaction of N-Acyliminium Ions Leading to Heterobicycles Arylated at Bridgehead Atom. Heteroat. Chem. 2014, $25,20$.

4. Stojanovi ć, M.; Markovi ć, R.; Kleinpeter, E.; BaranacStojanović, M., endo-Mode Cyclizations of Vinylogous N-Acyliminium Ions as a Route to the Synthesis of Condensed Thiazolidines. Tetrahedron 2011, 67, 9541.

5. Stojanovi ć, M.; Markovi ć, R., Synthesis of the First Thiazolidine-Condensed Five-, Six-, and Seven-Membered Heterocycles via Cyclization of Vinylogous N-Acyliminium Ions. Synlett 2009, 2009, 1997.

6. Stojanovic, M.; Markovic, R.; Kleinpeter, E.; BaranacStojanovic, M., Synthesis of Thiazolidine-Fused Heterocycles via exoMode Cyclizations of Vinylogous N-Acyliminium Ions. Org. Biomol. Chem. 2012, 10, 575.

7. Wang, Y.; Zhu, L.; Zhang, Y.; Hong, R., Bioinspired and Concise Synthesis of ( \pm )-Stemoamide. Angew. Chem., Int. Ed. 2011, $50,2787$.

8. Allin, S. M.; Gaskell, S. N.; Towler, J. M. R.; Page, P. C. B.; Saha, B.; McKenzie, M. J.; Martin, W. P., A New Asymmetric Synthesis of the Anti-Tumor Alkaloid (R)-(+)-Crispine A. J. Org. Chem. 2007, 72,8972 .

9. Dounay, A. B.; Overman, L. E.; Wrobleski, A. D., Sequential Catalytic Asymmetric Heck-Iminium Ion Cyclization: Enantioselective Total Synthesis of the Strychnos Alkaloid Minfiensine. J. Am. Chem. Soc. 2005, 127, 10186.

10. Saikia, A. K.; Indukuri, K.; Das, J., Stereoselective Synthesis of O-Tosyl Azabicyclic Derivatives via aza Prins Reaction of Endocyclic N-Acyliminium Ions: Application to the Total Synthesis of ( \pm )-epi-Indolizidine $167 B$ and 209D. Org. Biomol. Chem. 2014, 12, 7026 .

11. Hjelmgaard, T.; Søtofte, I.; Tanner, D., Total Synthesis of Pinnamine and Anatoxin-a via a Common Intermediate. A Caveat on the Anatoxin-a Endgame. J. Org. Chem. 2005, 70, 5688.

12. Hanessian, S.; Tremblay, M.; Petersen, J. F. W., The $N$ Acyloxyiminium Ion Aza-Prins Route to Octahydroindoles: Total Synthesis and Structural Confirmation of the Antithrombotic Marine Natural Product Oscillarin. J. Am. Chem. Soc. 2004, 126, 6064. 
13. Hanessian, S.; Tremblay, M., Tandem Functionalization of Nonactivated Alkenes and Alkynes in Intramolecular $\mathrm{N}$ Acyloxyiminium Ion Carbocyclization. Synthesis of 6-Substituted Hydroindole 2-Carboxylic Acids. Org. Lett. 2004, 6, 4683.

14. Peixoto, S.; Martin, M. T.; Crich, D.; Delpech, B.; Marazano, C., Concise Construction of Sarain A Core According to a Biosynthetic Proposal: Cyclization through an Intramolecular Mannich-Type Reaction Involving an Endocyclic N-Acyliminium Ion. Chem. - Eur. J. 2011, 17, 9907.

15. Peixoto, S.; Nguyen, T. M.; Crich, D.; Delpech, B.; Marazano, C., One-Pot Formation of Piperidine- and PyrrolidineSubstituted Pyridinium Salts via Addition of 5-Alkylaminopenta-2,4Dienals to N-Acyliminium Ions: Application to the Synthesis of ( \pm Nicotine and Analogs. Org. Lett. 2010, 12, 4760.

16. Padwa, A.; Danca, D. M.; Ginn, J. D.; Lynch, S. M., Application of the tandem thionium $/ \mathrm{N}$-acyliminium ion cascade toward heterocyclic synthesis. J. Braz. Chem. Soc. 2001, 12, 571-585.

17. Indukuri, K.; Unnava, R.; Deka, M. J.; Saikia, A. K., Stereoselective Synthesis of Amido and Phenyl Azabicyclic Derivatives via a Tandem Aza Prins-Ritter/Friedel-Crafts Type Reaction of Endocyclic N-Acyliminium Ions. J. Org. Chem. 2013, 78, 10629.

18. Chio, F. K. I.; Guesné, S. J. J.; Hassall, L.; McGuire, T.; Dobbs, A. P., Synthesis of Azabicycles via Cascade Aza-Prins Reactions: Accessing the Indolizidine and Quinolizidine Cores. J. Org. Chem. 2015, 80, 9868.

19. Koseki, Y.; Fujino, K.; Takeshita, A.; Sato, H.; Nagasaka, T., The Intramolecular Tandem Michael/Mannich-type Reaction of $\alpha, \beta$ -Unsaturated Carbonyl Compounds with Acyliminium Ions Provides Access to Chiral Indolizidines. Tetrahedron: Asymmetry 2007, 18, 1533.

20. Ballet, S.; Urbanczyk-Lipkowska, Z.; Tourwé, D., Synthesis of Substituted 4-Amino-2-Benzazepin-3-Ones via N-Acyliminium Ion Cyclizations. Synlett 2005, 2005, 2791.

21. Muratore, M. E.; Holloway, C. A.; Pilling, A. W.; Storer, R. I.; Trevitt, G.; Dixon, D. J., Enantioselective Brønsted Acid-Catalyzed N-Acyliminium Cyclization Cascades. J. Am. Chem. Soc. 2009, 131, 10796.

22. Zabaleta, N.; Uria, U.; Reyes, E.; Carrillo, L.; Vicario, J. L., Ion-pairing catalysis in the enantioselective addition of hydrazones to $\mathrm{N}$-acyldihydropyrrole derivatives. Chem. Commun. 2018, 54, 89058908 .

23. Dhanasekaran, S.; Suneja, A.; Bisai, V.; Singh, V. K., Approach to Isoindolinones, Isoquinolinones, and THIQs via Lewis Acid-Catalyzed Domino Strecker-Lactamization/Alkylations. Org. Lett. 2016, 18, 634-637.

24. Su ć, J.; Dokli, I.; Gredi čak, M., Chiral Brønsted acidcatalysed enantioselective synthesis of isoindolinone-derived N(acyl),S-acetals. Chem. Commun. 2016, 52, 2071-2074.

25. Raheem, I. T.; Thiara, P. S.; Peterson, E. A.; Jacobsen, E. N., Enantioselective Pictet-Spengler-Type Cyclizations of Hydroxylactams: H-Bond Donor Catalysis by Anion Binding. J. Am. Chem. Soc. 2007, 129, 13404.

26. Taylor, M. S.; Tokunaga, N.; Jacobsen, E. N., Enantioselective Thiourea-Catalyzed Acyl-Mannich Reactions of Isoquinolines. Angew. Chem., Int. Ed. 2005, 44, 6700.

27. Peterson, E. A.; Jacobsen, E. N., Enantioselective, ThioureaCatalyzed Intermolecular Addition of Indoles to Cyclic N-Acyl Iminium Ions. Angew. Chem., Int. Ed. 2009, 48, 6328.

28. Klausen, R. S.; Jacobsen, E. N., Weak Brønsted AcidThiourea Co-catalysis: Enantioselective, Catalytic Protio-PictetSpengler Reactions. Org. Lett. 2009, 11, 887.

29. Maity, A. K.; Roy, S., Multimetallic Iridium-Tin (Ir-Sn3) Catalyst in N-Acyliminium Ion Chemistry: Synthesis of 3-Substituted Isoindolinones via Intra- and Intermolecular Amidoalkylation Reaction. Adv. Synth. Catal. 2014, 356, 2627-2642.

30. Maity, A. K.; Roy, S., A Multimetallic Piano-Stool Ir-Sn3 Catalyst for Nucleophilic Substitution Reaction of $\gamma$-Hydroxy Lactams through N-Acyliminium Ions. J. Org. Chem. 2012, 77, 2935-2941.

31. Ben Othman, R.; Affani, R.; Tranchant, M.-J.; Antoniotti, S.; Dalla, V.; Duñach, E., N-Acyliminium Ion Chemistry: Highly Efficient and Versatile Carbon-Carbon Bond Formation by Nucleophilic Substitution of Hydroxy Groups Catalyzed by Sn(NTf2)4. Angew. Chem. Int. Ed. 2010, 49, 776-780.
32. Nilson, M. G.; Funk, R. L., Generation of N-Acyliminium Ions via Intramolecular Conjugate Addition Reactions: A Strategy for the Total Synthesis of Nakadomarin A. Org. Lett. 2006, 8, 3833.

33. Nilson, M. G.; Funk, R. L., Total Synthesis of (-)Nakadomarin A. Org. Lett. 2010, 12, 4912.

34. Abe, H.; Aoyagi, S.; Kibayashi, C., Total Synthesis of the Tricyclic Marine Alkaloids (-)-Lepadiformine, (+)-Cylindricine C, and (-)-Fasicularin via a Common Intermediate Formed by Formic Acid-Induced Intramolecular Conjugate Azaspirocyclization. J. Am. Chem. Soc. 2005, 127, 1473.

35. Jones, A. M.; Banks, C. E., The Shono-type electroorganic oxidation of unfunctionalised amides. Carbon-carbon bond formation via electrogenerated N-acyliminium ions. Beilstein. J. Org. Chem. 2014, 10, 3056-3072.

36. Britten, T. K.; McLaughlin, M. G., Brønsted Acid Catalyzed Peterson Olefinations. J. Org. Chem. 2020, 85, 301-305.

37. Basson, A. J.; McLaughlin, M. G., Functionalisation of isoindolinones via a calcium catalysed Hosomi-Sakurai allylation. Chem. Commun. 2019, 55, 8317-8320.

38. Kiely-Collins, H. J.; Sechi, I.; Brennan, P. E.; McLaughlin, M. G., Mild, calcium catalysed Beckmann rearrangements. Chem. Commun. 2018, 54, 654-657.

39. Harder, S., From Limestone to Catalysis: Application of Calcium Compounds as Homogeneous Catalysts. Chem. Rev. 2010, 110, 3852-3876.

40. Begouin, J. M.; Niggemann, M., Calcium-based Lewis acid catalysts. Chem. Eur. J. 2013, 19, 8030-41.

41. Fu, L.; Niggemann, M., Calcium-Catalyzed Carboarylation of Alkynes. Chem. Eur. J. 2015, 21, 6367-6370.

42. Meyer, V. J.; Niggemann, M., Calcium-Catalyzed Direct Coupling of Alcohols with Organosilanes. Eur. J. Org. Chem. 2011, 2011, 3671-3674.

43. Gao, S.; Stopka, T.; Niggemann, M., Calcium-Catalyzed Dynamic Multicomponent Reaction. Org. Lett. 2015, 17, 5080-5083.

44. Meyer, V. J.; Ascheberg, C.; Niggemann, M., CalciumCatalyzed Formal $[2+2+2]$ Cycloaddition. Chem. Eur. J. 2015, 21, 6371-6374.

45. Meike, N.; J., M. M., Calcium-Catalyzed Friedel-Crafts Alkylation at Room Temperature. Angew. Chem. Int. Ed. 2010, 49, 3684-3687.

46. Stopka, T.; Niggemann, M., Cyclopentanone as a CationStabilizing Electron-Pair Donor in the Calcium-Catalyzed Intermolecular Carbohydroxylation of Alkynes. Org. Lett. 2015, 17, $1437-1440$.

47. Begouin, J.-M.; Capitta, F.; Wu, X.; Niggemann, M., Diastereoselective Synthesis of Indanes and Tetralins via Intramolecular Friedel-Crafts Reaction. Org. Lett. 2013, 15, 13701373 .

48. Crimmin, M. R.; Barrett, A. G. M.; Hill, M. S.; MacDougall, D. J.; Mahon, M. F.; Procopiou, P. A., Chem.-Eur. J. 2008, 14, 11292.

49. Barrett, A. G. M.; Crimmin, M. R.; Hill, M. S.; Hitchcock, P. B.; Kociok-Köhn, G.; Procopiou, P. A., Triazenide Complexes of the Heavier Alkaline Earths: Synthesis, Characterization, And Suitability for Hydroamination Catalysis. Inorg. Chem. 2008, 47, 7366.

50. Barrett, A. G. M.; Casely, I. J.; Crimmin, M. R.; Hill, M. S.; Lachs, J. R.; Mahon, M. F.; Procopiou, P. A., $\beta$-Diketiminato Calcium and Magnesium Amides; Model Complexes for Hydroamination Catalysis. Inorg. Chem. 2009, 48, 4445.

51. Crimmin, M. R.; Arrowsmith, M.; Barrett, A. G. M.; Casely, I. J.; Hill, M. S.; Procopiou, P. A., Intramolecular Hydroamination of Aminoalkenes by Calcium and Magnesium Complexes: A Synthetic and Mechanistic Study. J. Am. Chem. Soc. 2009, 131, 9670.

52. Crimmin, M. R.; Barrett, A. G. M.; Hill, M. S.; Hitchcock, P. B.; Procopiou, P. A., Calcium-Catalyzed Intermolecular Hydrophosphination. Organometallics 2007, 26, 2953.

53. Barrett, A. G. M.; Brinkmann, C.; Crimmin, M. R.; Hill, M. S.; Hunt, P.; Procopiou, P. A., Heavier Group 2 Metals and Intermolecular Hydroamination: A Computational and Synthetic Assessment. J. Am. Chem. Soc. 2009, 131, 12906.

54. Lachs, J. R.; Barrett, A. G. M.; Crimmin, M. R.; KociokKöhn, G.; Hill, M. S.; Mahon, M. F.; Procopiou, P. A., Heavier Group-2-Element Catalyzed Hydroamination of Carbodiimides. Eur. J. Inorg. Chem. 2008, 26, 4173. 
55. Barrett, A. G. M.; Crimmin, M. R.; Hill, M. S.; Hitchcock, P. B.; Lomas, S. L.; Mahon, M. F.; Procopiou, P.; Suntharalingam, K., $\beta$-Diketiminato Calcium Acetylides: Synthesis, Solution Dimerization, and Catalytic Carbon-Carbon Bond Formation. Organometallics 2008, 27, 6300 .

56. Crimmin, M. R.; Barrett, A. G. M.; Hill, M. S.; Hitchcock, P. B.; Procopiou, P. A., Bis(trimethylsilyl)methyl Derivatives of Calcium, Strontium and Barium: Potentially Useful Dialkyls of the Heavy Alkaline Earth Elements. Organometallics 2008, 27, 497.

57. Barrett, A. G. M.; Boorman, T. C.; Crimmin, M. R.; Hill, M. S.; Kociok-Köhn, G.; Procopiou, P., Heavier group 2 elementcatalysed hydroamination of isocyanates. Chem. Commun. 2008, 5206.

58. van Nguyen, H.; Matsubara, R.; Kobayashi, S., Addition Reactions of Sulfonylimidates with Imines Catalyzed by Alkaline Earth Metals. Angew. Chem., Int. Ed. 2009, 48, 5927.

59. Crimmin, M. R.; Barret, A. G. M.; Hill, M. S.; Procopiou, P. A., Heavier Alkaline Earth Amides as Catalysts for the Tischenko Reaction. Org. Lett. 2007, 9, 331.

60. Barrett, A. G. M.; Crimmin, M. R.; Hill, M. S.; Hitchcock, P. B.; Thomas, S. L.; Procopiou, P. A.; Suntharalingam, K., Catalytic 2,3,4-hexatriene formation by terminal alkyne coupling at calcium. Chem. Commun. 2009, 2299.

61. Qi, C.; Gandon, V.; Leb œuf, D., Calcium(II)-Catalyzed Alkenylation of $\mathrm{N}$-Acyliminiums and Related Ions with Vinylboronic Acids. Adv. Synth. Catal. 2017, 359, 2671-2675.

62. Parsons, P. J.; Jones, D. R.; Walsh, L. J.; Allen, L. A. T.; Onwubiko, A.; Preece, L.; Board, J.; White, A. J. P., An Approach to the Core of Lactonamycin. Org. Lett. 2017, 19, 2533.

63. Flores, B.; Molinski, T. F., Assembly of the Isoindolinone Core of Muironolide a by Asymmetric Intramolecular Diels-Alder Cycloaddition. Org. Lett. 2011, 13, 3932.

64. Augner, D.; Schmalz, H. G., Biomimetic Synthesis of Isoindolinones Related to the Marilines. Synlett 2015, 26, 1395.
65. Hu, S.; Yuan, L.; Yan, H.; Li, Z., Design, Synthesis and Biological Evaluation of Lenalidomide Derivatives as Tumor Angiogenesis Inhibitor. Bioorg. Med. Chem. Lett. 2017, 27, 4075.

66. Hardcastle, I. R.; Ahmed, S. U.; Atkins, H.; Farnie, G.; Golding, B. T.; Griffin, R. J.; Guyenne, S.; Hutton, C.; Källblad, P.; Kemp, S. J.; Kitching, M. S.; Newell, D. R.; Norbedo, S.; Northen, J. S.; Reid, R. J.; Saravanan, K.; Willems, H. M. G.; Lunec, J., SmallMolecule Inhibitors of the MDM2-p53 Protein-Protein Interaction Based on an Isoindolinone Scaffold. J. Med. Chem. 2006, 49, 62096221.

67. Mertens, A.; Zilch, H.; Koenig, B.; Schaefer, W.; Poll, T.; Kampe, W.; Seidel, H.; Leser, U.; Leinert, H., Selective non-nucleoside HIV-1 reverse transcriptase inhibitors. New 2,3-dihydrothiazolo[2,3a]isoindol-5(9bH)-ones and related compounds with anti-HIV-1 activity. J. Med. Chem. 1993, 36, 2526-2535.

68. Richa Kaur, B., Isoindole Derivatives: Propitious Anticancer Structural Motifs. Current Topics in Medicinal Chemistry 2017, 17, 189-207.

69. Georg, G. I., Recent advances in the chemistry and biology of $\beta$-lactams and $\beta$-lactam antibiotics. Bioorg. Med. Chem. Lett. 1993, 3, 2157.

70. Unhale, R. A.; Molleti, N.; Rana, N. K.; Dhanasekaran, S.; Bhandary, S.; Singh, V. K., Chiral phosphoric acid catalyzed enantioselective addition of thiols to in situ generated ketimines: Synthesis of N,S-ketals. Tetrahedron Lett. 2017, 58, 145-151.

71. Scherlach, K.; Schuemann, J.; Dahse, H.-M.; Hertweck, C., Aspernidine $A$ and $B$, prenylated isoindolinone alkaloids from the model fungus Aspergillus nidulans. The Journal of Antibiotics 2010, 63, 375-377.

72. Zhou, H.; Sun, X.; Li, N.; Che, Q.; Zhu, T.; Gu, Q.; Li, D., Isoindolone-Containing Meroperpenoids from the Endophytic Fungus Emericella nidulans HDN12-249. Organic Letters 2016, 18, 46704673. 